Aliang Xia*

\title{
Multiplicity and concentration results for magnetic relativistic Schrödinger equations
}

https://doi.org/10.1515/anona-2020-0044

Received February 18, 2019; accepted October 4, 2019.

Abstract: In this paper, we consider the following magnetic pseudo-relativistic Schrödinger equation

$$
\sqrt{\left(\frac{\varepsilon}{i} \nabla-A(x)\right)^{2}+m^{2}} u+V(x) u=f(|u|) u \text { in } \mathbb{R}^{N},
$$

where $\varepsilon>0$ is a parameter, $m>0, N \geq 1, V: \mathbb{R}^{N} \rightarrow \mathbb{R}$ is a continuous scalar potential satisfies $V(x) \geq$ $-V_{0}>-m$ for any $x \in \mathbb{R}^{N}$ and $f: \mathbb{R}^{N} \rightarrow \mathbb{R}$ is a continuous function. Under a local condition imposed on the potential $V$, we discuss the number of nontrivial solutions with the topology of the set where the potential attains its minimum. We proof our results via variational methods, penalization techniques and LjusternikSchnirelmann theory.

Keywords: magnetic pseudo-relativistic operators; Nehari manifold; Ljusternik-Schnirelmann theory

MSC: 35J10; 35Q40; 35S05; 35J50

\section{Introduction and statement of main results}

In this paper, we consider the mean field limit of a quantum system with rest mass $m>0$ in the presence of a magnetic vector potential $A(x)$ and an electric potential $V(x)$. More precise, we focus our attention on the following time-depend pseudo-relativistic magnetic Schrödinger equation

$$
i \varepsilon \frac{\partial \psi}{\partial t}=\left(\sqrt{\left(\frac{\varepsilon}{i} \nabla-A(x)\right)^{2}+m^{2}}-m\right) \psi+V(x) \psi-f(|\psi|) \psi \text { in } \mathbb{R}^{N},
$$

where $\varepsilon>0$ is a small positive constant, $i$ is the imaginary unit, $m>0, N \geq 1, \psi: \mathbb{R}^{N} \times \mathbb{R} \rightarrow \mathbb{C}$ is a wave field, $A: \mathbb{R}^{N} \rightarrow \mathbb{R}^{N}$ is a continuous vector potential, $V: \mathbb{R}^{N} \rightarrow \mathbb{R}$ is an external continuous scalar potential and function $f: \mathbb{R}^{N} \rightarrow \mathbb{R}$. The magnetic relativistic Schrödinger operator relate to the classical relativistic Hamiltonian symbol in Fourier variables

$$
\sqrt{(\xi-A(x))^{2}+m^{2}}+V(x), \quad(\xi, x) \in \mathbb{R}^{N} \times \mathbb{R}^{N},
$$

which is the sum of the kinetic energy term. This operator is known as a spinless particle in electromagnetic fields where we ignore quantum field theoretic effect like particles creation and annihilation but should take relativistic effect into consideration, see [1, 2]. We should remark that there are three type of relativistic Hamiltonian depending on how we quantize the kinetic energy symbol $\sqrt{(\xi-A(x))^{2}+m^{2}}$. The first two quantized operators defined by mean formulas, that is, for any function $\varphi \in C_{0}^{\infty}\left(\mathbb{R}^{N}, \mathbb{C}\right)$,

$$
\mathcal{H}_{A}^{1} \varphi(x):=\frac{1}{4 \pi^{2}} \int_{\mathbb{R}^{2 N}} e^{i(x-y) \cdot \xi} \sqrt{\left(\xi-A\left(\frac{x+y}{2}\right)\right) m^{2}} \varphi(y) d y d \xi
$$

*Corresponding Author: Aliang Xia, Department of Mathematics, Jiangxi Normal University, Nanchang, Jiangxi 330022, P. R. China, E-mail: aliang_xia@jxnu.edu.cn 
and

$$
\mathcal{H}_{A}^{2} \varphi(x):=\frac{1}{4 \pi^{2}} \int_{\mathbb{R}^{2 N}} e^{i(x-y) \cdot \xi} \sqrt{\left(\xi-\int_{0}^{1} A((1-\theta) x+\theta y) d \theta\right) m^{2} \varphi(y) d y d \xi .}
$$

We note that the Weyl pseudo-differential operator $\mathcal{H}_{A}^{1}$ is not covariant under gauge transformations, that is, $\mathcal{H}_{A+\nabla \phi}^{1} \neq e^{i \phi} \mathcal{H}_{A}^{1} e^{-i \phi}$. The operator $\mathcal{H}_{A}^{2}$ is a modification of operator $\mathcal{H}_{A}^{1}$, which is gauge covariant, see [3]. The third quantized $\mathcal{H}_{A}^{3}$ is the square of the nonnegative selfadjoint operator $(-i \nabla-A(x))^{2}+m^{2}$, that is,

$$
\mathcal{H}_{A}^{3}=\sqrt{(-i \nabla-A(x))^{2}+m^{2}} .
$$

The operator $\mathcal{H}_{A}^{3}$ is gauge covariant and is used in the description of the stability of the matter in relativistic quantum mechanics, see for example [4, 5]. All three quantized operators are different from one another (see $[1,6])$. As we know that they coincide if $A(x)$ is linear, that is, $A(x)=A \cdot x$, with $A$ is a real symmetric constant matrix, see [1]. Particularly, this holds for constant magnetic field when $N=3$, that is, $B=\nabla \times A$ is constant.

A solution of the form $\psi(x, t)=e^{i E t / \varepsilon} u(x)$ is called a solitary wave. Then $\psi(x, t)$ is a solution of (1.1) if and only if the function $u$ satisfies

$$
\sqrt{\left(\frac{\varepsilon}{i} \nabla-A(x)\right)^{2}+m^{2}} u+V(x) u=f(|u|) u \text { in } \mathbb{R}^{N},
$$

where we write $V$ instead of $V+(E-m)$ for simplicity.

Recently, Cingolani and Secchi in [7] studied the interwining solutions of magnetic relativistic Hartree type equations, that is,

$$
\sqrt{(-i \nabla-A(x))^{2}+m^{2}} u+V(x) u=\left(|x|^{\alpha-N} \star|u|^{p}\right)|u|^{p-2} u \text { in } \mathbb{R}^{N},
$$

where $2 \leq p<2 N /(N-1)$ and $(N-1) p-N<\alpha<N$. Their proofs are based on the variational methods and Caffarelli and Silvestre's type extension (see [8]) for pseudo-relativistic magnetic Schrödinger operator $\sqrt{(-i \nabla-A(x))^{2}+m^{2}}+V(x)$ when $A(x)$ is uniformly bounded or linear in $x$. If $N=3$ and $\alpha=p=2$, which corresponds to the Coulomb kernel, equation (1.3) is often referred to a boson star in astrophysics, see for example $[9,10]$. If also assume $A \equiv 0$ and $V(x)=-m$, equation (1.3) is reduced to the classical pseudorelativistic Hartree equation which introduced by Lieb and Yau [11], see also [12-14] and references therein.

In the literature, the existence of standing waves solutions to nonlinear magnetic Schrödinger equation

$$
\left(\frac{\varepsilon}{i} \nabla-A(x)\right)^{2}+V(x) u=f(x, u), \quad \text { in } \mathbb{R}^{N}
$$

has been first studied by Lions and Esteban [15], for $\varepsilon>0$ fixed and special classes of magnetic fields. They have found existence results by solving appropriate minimization problems and concentration-compactness method for the corresponding energy functional in the cases $N=2$ and 3. Lately, Kurata [16] studied the existence of a least energy solution of (1.3) under a condition relating $V(x)$ and $A(x)$; Cingolani [17] and Alves et al. [18] investigated the multiplicity results of (1.3) by applying the Ljusternik-Schnirelmann theory. We refer readers to [17, 19-21] and references therein for other results about nonlinear magnetic Schrödinger equation.

For the nonlocal magnetic Schrödinger equations have been investigated recently. The fractional magnetic Laplacian is defined by

$$
(-\Delta)_{A}^{S} u(x):=\lim _{r \rightarrow 0} \int_{B_{r}^{c}(x)} \frac{u(x)-e^{i(x-y) \cdot A\left(\frac{x+y}{2}\right)} u(y)}{|x-y|^{N+2 s}} d y, \quad s \in(0,1),
$$

which is deduced from the magnetic operator $\mathcal{H}_{A}^{1}$ for smooth functions $u$. In quantum mechanics, when $\varepsilon \rightarrow 0$, the existence and concentration of solution is of particular importance. The existence and concentration results for fractional magnetic Schrödinger equations were studied by Ambrosio and d'Avenia [22], Fiscella, Pinamonti and Vecchi [23], Zhang, Squassina and Xia [24], Mao and Xia [25]. We also refer to d'Avenia 
and Squassina [26] for the existence of ground states and other useful estimates. Lastly, for the existence and multiplicity results of semilinear or quasilinear Schrödinger equations, we refer readers to [27-29] and references therein.

Motivated by the about results, in this paper we deal with multiplicity and concentration results of the more general class of pseudo-relativistic magnetic Schrödinger equation (1.2) . In what follows, on potentials we assume that

(A) $A: \mathbb{R}^{N} \rightarrow \mathbb{R}$ is a continuous functions and uniformly bounded.

(V1) $V: \mathbb{R}^{N} \rightarrow \mathbb{R}$ is a continuous functions satisfies $V+V_{0} \geq 0$ for some $V_{0} \in(0, m)$ and every $x \in \mathbb{R}^{N}$.

(V2)There is a bounded open set $\mathcal{O} \subset \mathbb{R}^{N}$ such that

$$
\min _{\partial \mathcal{O}} V>-V_{0}
$$

and $M=\left\{x \in \mathcal{O}: V(x)=-V_{0}\right\} \neq \emptyset$.

Also, we suppose continuous function $f$ satisfying

(f1) $f(s)=0$, for all $s \leq 0$ and $f(s)=o(s)$ as $s \rightarrow 0^{+}$.

(f2) There exists constants $q, \sigma \in\left(2,2^{\sharp}\right)$ where $2^{\sharp}:=\frac{2 N}{N-1}$ if $N \geq 2$ and $2^{\sharp}:=\infty$ if $N=1, C_{0}>0$ such that $f(s) \geq C_{0} s^{q-2}$ for all $s>0$ and

$$
\lim _{s \rightarrow+\infty} \frac{f(s)}{s^{\sigma-2}}=0 .
$$

(f3) There exists a constant $\theta \in\left(2,2^{\sharp}\right)$ such that

$$
0<\theta F(s):=\theta \int_{0}^{s} f(\tau) \tau d \tau \leq f(s) s^{2} \quad \text { for all } s>0 .
$$

(f4) The function $f(s)$ is increasing in $(0,+\infty)$.

We shall establish a relation between the number of solutions of (1.2) and the topology of the set $M$. In order to make a precise statement let us recall that, for any closed subset $Y$ of a topological space $X$, the LjusternikSchnirelmann category of $Y$ in $X, \operatorname{cat}_{X}(Y)$, stands for the least number of closed and contractible sets in $X$ which cover $Y$.

The main result of this article is

Theorem 1.1. Assume that $(A),(V 1)-(V 2)$ and $(f 1)-(f 4)$ hold. Then for and $\delta>0$ such that

$$
M_{\delta}=\left\{x \in \mathbb{R}^{N}: \operatorname{dist}(x, M) \leq \delta\right\} \subset \mathcal{O},
$$

there exists $\varepsilon_{\delta}>0$ such that problem (1.2) has at least cat $M_{M_{\delta}}(M)$ solutions provides $\varepsilon \in\left(0, \varepsilon_{\delta}\right)$. Moreover, if $u_{\varepsilon}$ denotes one of these solutions and $\eta_{\varepsilon} \in \mathbb{R}^{N}$ its global maximum, then

$$
\lim _{\varepsilon \rightarrow 0} V\left(\eta_{\varepsilon}\right)=-V_{0} .
$$

It should be pointed out that we only assume the potential $V(x)$ satisfies local conditions $(V 1)-(V 2)$ and no information on the behavior of the potential $V(x)$ at infinity, so we will use the penalization method introduced by del Pino and Felmer [30] rather than minimax theorem to prove our main results. It is worthwhile to remark that in the arguments developed in [30], one of the key points is the existence of estimates involving the $L^{\infty}$-bounds of the modified problem. Here we obtain the desired $L^{\infty}$-bounds via Moser's iteration method (see [31]) instead of Kato's inequality. Moreover, we get the multiplicity results by Ljusternik-Schnirelmann theory (see [32]). As far as we known, this is the first time that penalization scheme and topological arguments are combined to get multiple solutions for magnetic pseudo-relativistic equations.

We also remark that we assume the nonlinearity term $f$ is only continuous, so we can not use the standard arguments on the Nehari manifold. To overcome the non-differentiability for the Nehari manifold, we shall 
use some variants of critical point theorems from Szulkin and Weth [32]. This idea has been used extensively for nonlocal elliptic problems, see for example [33, 34].

Our proof based on the Caffarelli and Silvestre's type extension (see [8]) for pseudo-relativistic magnetic Schrödinger operator $\sqrt{(-i \nabla-A(x))^{2}+m^{2}}+V(x)$ when $A(x)$ is uniformly bounded, which is prove by Cingolani and Secchi in [7]. However, some difficulties appear since the nonlinearity is on the boundary. In particular, in order to obtain the $L^{\infty}$-bounds in Section 4 we will establish an inverse Hölder inequality for $y(w)=u$ and we my iterate the inequality for $y(w)$.

This paper is organized as follows. In section 2, we present the variational setting of the original and the extended variables problems, and we modify the original problem. We also prove the Palais-Smale condition for the modified functional and obtain some tools which are useful to establish a multiplicity result. In section 3 , we study the autonomous problem associated which allow us to prove the modified problem has multiple solutions. Finally, we prove Theorem 1.1 via Morse iteration method.

\section{Extension and modified problem}

In this paper, we will systematically consider spaces of complex-valued functions. Precisely, the $L^{2}\left(\mathbb{R}^{N}, \mathbb{C}\right)$ space will be endowed with the real scalar product

$$
\langle v, w\rangle_{L^{2}}=\Re \int_{\mathbb{R}^{N}} v(x) \overline{w(x)} d x .
$$

In what follows, we will write $|\cdot| p$ for the norm in $L^{p}\left(\mathbb{R}^{N}\right)$ and $\|\cdot\|_{p}$ for the norm in $L^{p}\left(\mathbb{R}_{+}^{N+1}\right)$. Moreover, for any $w \in H^{1}\left(\mathbb{R}_{+}^{N+1}, \mathbb{C}\right)$, we denote

$$
\|w\|_{H^{1}}^{2}=\int_{\mathbb{R}_{+}^{N+1}}\left(|\nabla w|^{2}+|w|^{2}\right) d x d y, \quad \text { for }(x, y) \in \mathbb{R}^{N} \times \mathbb{R}_{+} .
$$

Let $\tilde{A}(x, y)=(A(x), 0): \mathbb{R}_{+}^{N+1} \rightarrow \mathbb{R}^{N+1}$ be the trivial lifting of a vector field $A(x): \mathbb{R}^{N} \rightarrow \mathbb{R}^{N}$ for every $(x, y) \in \mathbb{R}_{+}^{N+1}$. Then, we define the magnetic Sobolev spaces on the half-space $H_{\tilde{A}}^{1}\left(\mathbb{R}_{+}^{N+1}, \mathbb{C}\right)$ as

$$
H_{\tilde{A}}^{1}\left(\mathbb{R}_{+}^{N+1}, \mathbb{C}\right):=\left\{w \in L^{2}\left(\mathbb{R}_{+}^{N+1}, \mathbb{C}\right):\left(\frac{\nabla}{i}-\tilde{A}\right) w \in L^{2}\left(\mathbb{R}_{+}^{N+1}\right)\right\},
$$

which endowed with the norm

$$
\|w\|_{H_{\tilde{A}}^{1}}=\left(\int_{\mathbb{R}_{+}^{N+1}}\left|\left(\frac{\nabla}{i}-\tilde{A}\right) w\right|^{2} d x d y+\|w\|_{L^{2}}^{2}\right)^{\frac{1}{2}}
$$

and the scalar product

$$
\langle v, w\rangle_{H_{\tilde{A}}^{1}}:=\Re \int_{\mathbb{R}_{+}^{N+1}} \nabla_{\tilde{A}} v \overline{\nabla_{\tilde{A}} w}+v \bar{w} d x d y,
$$

where

$$
\nabla_{\tilde{A}}=\frac{\nabla}{i}-\tilde{A} .
$$

For simplicity, we will write $H_{\tilde{A}}^{1}\left(\mathbb{R}_{+}^{N+1}, \mathbb{C}\right)$ and $\|w\|_{H_{\tilde{A}}^{1}}$ as $H_{A}^{1}\left(\mathbb{R}_{+}^{N+1}, \mathbb{C}\right)$ and $\|w\|_{H_{A}^{1}}$ respectively.

Next, we recall the following result about trace in magnetic Sobolev space operator which proved in [7].

Proposition 2.1. Suppose that $A$ is bounded. Then a surjective continuous linear map $y: H_{A}^{1}\left(\mathbb{R}^{N+1}, \mathbb{C}\right) \rightarrow$ $H_{A}^{1 / 2}\left(\mathbb{R}^{N}, \mathbb{C}\right)$ is defined so that $y(w)=\left.w\right|_{\mathbb{R}^{N} \times\{0\}}$ for every $w \in C^{1}\left(\mathbb{R}^{N+1}, \mathbb{C}\right)$, where

$$
H_{A}^{1 / 2}\left(\mathbb{R}^{N}, \mathbb{C}\right)=\left\{u \in L^{2}\left(\mathbb{R}^{N}, \mathbb{C}\right):|u|_{L^{2}}^{2}+\int_{\mathbb{R}^{2 N}} \frac{\left|u(x)-e^{i(x-y) \cdot A\left(\frac{x+y}{2}\right)} u(y)\right|^{2}}{|x-y|^{N+1}} d x d y<+\infty\right\} .
$$


This result allows us to generalized the well-known Dirichlet-to-Neumann extension for fractional Laplacian to the magnetic pseudo-relativistic operator. Letting $\Delta_{\tilde{A}}=-\nabla_{\tilde{A}}^{2}=\Delta_{A}+\frac{\partial^{2}}{\partial y^{2}}$ where

$$
\Delta_{A}=\Delta_{x} u-2 i A(x) \cdot \nabla_{x} u-i \operatorname{div}(A(x))-|A(x)|^{2},
$$

then Cingonali and Secchi in [7] showed that

Proposition 2.2. For $u \in H^{1 / 2}\left(\mathbb{R}^{N}, \mathbb{C}\right)$, then there exists one and only one function $w \in H_{A}^{1}\left(\mathbb{R}_{+}^{N+1}, \mathbb{C}\right)$ such that

$$
\begin{cases}-\Delta_{\tilde{A}} w+m^{2} w=0 & \text { in } \mathbb{R}_{+}^{N+1}, \\ w=u & \text { in } \mathbb{R}^{N}=\partial \mathbb{R}_{+}^{N+1} .\end{cases}
$$

We remark that the key point of the proof of Proposition 2.2 is to show that magnetic Sobolev spaces $H_{A}^{1}\left(\mathbb{R}_{+}^{N+1}, \mathbb{C}\right)$ and $H_{A}^{1 / 2}\left(\mathbb{R}^{N}, \mathbb{C}\right)$ are equivalent to $H^{1}\left(\mathbb{R}_{+}^{N+1}, \mathbb{C}\right)$ and $H^{1 / 2}\left(\mathbb{R}^{N}, \mathbb{C}\right)$ respectively when $A(x)$ is bounded. Therefore, the existence of trace operator follows immediately from the standard theory of Sobolev traces in non-magnetic spaces. Hence, by Proposition 2.1, we deduce that the embeddings

$$
H_{A}^{1}\left(\mathbb{R}_{+}^{N+1}, \mathbb{C}\right) \hookrightarrow L^{p}\left(\mathbb{R}^{N}\right) \quad \text { for } p \in\left[2,2^{\sharp}\right]
$$

are continuous when $A$ is uniformly bounded.

By Proposition 2.2, we know that every function $w \in H_{A}^{1}\left(\mathbb{R}_{+}^{N+1}, \mathbb{C}\right)$ possesses a trace $y(w) \in H^{1 / 2}\left(\mathbb{R}^{N}, \mathbb{C}\right)$. Moreover, the following inequality holds

$$
|y(w)|_{p}^{p} \leq p\|w\|_{2(p-1)}^{p-1}\left\|\frac{\partial w}{\partial y}\right\|_{2}
$$

provides $2 \leq p \leq 2^{\sharp}$. For the proofs of (2.5), one can find in [7].

It is easy to see that problem (1.2) is equivalent, after a change of variable, to the following one

$$
\sqrt{\left(-i \nabla-A_{\varepsilon}(x)\right)^{2}+m^{2}} u+V_{\varepsilon}(x) u=f(|u|) u \text { in } \mathbb{R}^{N},
$$

where $A_{\varepsilon}(x)=A(\varepsilon x)$ and $V_{\varepsilon}(x)=V(\varepsilon x)$. Once we obtain a solution of (2.6), then the function $\tilde{u}_{\varepsilon}(x)=u_{\varepsilon}(x / \varepsilon)$ is a solution of (1.2). Moreover, the maximum $\zeta_{\varepsilon}$ of $\tilde{u}_{\varepsilon}$ is related to the maximum point $z_{\varepsilon}$ of $u_{\varepsilon}$ by $\zeta_{\varepsilon}=\varepsilon z_{\varepsilon}$.

By applying Proposition 2.2, we are interested to the study of the relativistic magnetic nonlocal equation

$$
\begin{cases}-\Delta_{\tilde{A}_{\varepsilon}} w+m^{2} w=0 & \text { in } \mathbb{R}_{+}^{N+1}, \\ -\frac{\partial w}{\partial y}=-V_{\varepsilon}(x) w+f(|w|) w & \text { in } \mathbb{R}^{N}=\partial \mathbb{R}_{+}^{N+1},\end{cases}
$$

where $\tilde{A}_{\varepsilon}=\left(A_{\varepsilon}, 0\right)$. We also observe that, for every $m>0$, (2.5) implies that

$$
\begin{aligned}
\int_{\mathbb{R}^{N}}|y(w)|^{2} d x & \leq m \int_{\mathbb{R}_{+}^{N+1}}|w|^{2} d x d y+\frac{1}{m} \int_{\mathbb{R}_{+}^{N+1}}\left|\frac{\partial w}{\partial y}\right|^{2} d x d y \\
& \leq m \int_{\mathbb{R}_{+}^{N+1}}|w|^{2} d x d y+\frac{1}{m} \int_{\mathbb{R}_{+}^{N+1}}\left(\left|\left(-i \nabla_{x}-A_{\varepsilon}(x)\right) w\right|^{2}+\left|\frac{\partial w}{\partial y}\right|^{2}\right) d x d y .
\end{aligned}
$$

Since there is no information about the infinity of $V(x)$, we adapt the penalization method introduced by del Pino and Felmer [30] to establish the multiplicity results. Let $K>\frac{2 V_{0}}{m-V_{0}}, a>0$ such that $f(a)=\frac{V_{0}}{K}$ where $V_{0}$ given in (V1). Define

$$
\tilde{f}(s)= \begin{cases}f(s) & \text { if } s \leq a, \\ \frac{V_{0}}{K} & \text { if } s>a,\end{cases}
$$

and

$$
g(x, s)=\chi_{\mathcal{O}}(x) f(s)+\left(1-\chi_{\mathcal{O}}(x)\right) \tilde{f}(s)
$$

where $\chi_{\mathcal{O}}(x)$ is the characteristic of set $\mathcal{O}$. By the assumptions $(f 1)-(f 4)$, it is easy to check that $g$ is a Carathéodory function and satisfies 
(g1) $\lim _{s \rightarrow 0^{+}} g(x, s)=0$.

(g2) $g(x, s) \leq f(s)$ for all $x \in \mathbb{R}^{N}, s>0$.

(g3) (i) $0<\theta G(x, s):=\theta \int_{0}^{s} g(x, \tau) \tau d \tau \leq g(x, s) s^{2} \quad$ for all $x \in \mathcal{O}, s>0$;

(ii) $0 \leq 2 G(x, s)<g(x, s) s^{2} \leq \frac{V_{0}}{K} s^{2}$ for all $x \in \mathbb{R}^{N} \backslash \mathcal{O}$ and $s>0$.

(g4)For each $x \in \mathcal{O}$, the function $s \mapsto g(x, s)$ is increasing in $(0,+\infty)$, and for each $x \in \mathcal{O}^{c}$, the function $s \mapsto g(x, s)$ is increasing in $(0, a)$.

Therefore, we study the auxiliary problem

$$
\begin{cases}-\Delta_{\tilde{A}_{\varepsilon}} w+m^{2} w=0 & \text { in } \mathbb{R}_{+}^{N+1}, \\ -\frac{\partial w}{\partial y}=-V_{\varepsilon}(x) w+g_{\varepsilon}(x,|w|) w & \text { in } \mathbb{R}^{N}=\partial \mathbb{R}_{+}^{N+1},\end{cases}
$$

where $g_{\varepsilon}(x, w)=g(\varepsilon x, w)$. Note that solution of (2.10) with $w(x) \leq a$ for each $x \in \mathcal{O}_{\varepsilon}$ are also the solution of (2.7), where $\mathcal{O}_{\varepsilon}=\left\{x \in \mathbb{R}^{N}: \varepsilon x \in \mathcal{O}\right\}$.

Consider the Euler-Lagrange functional associated to (2.10) given by

$$
\begin{aligned}
\mathcal{J}_{\mathcal{E}}(w)= & \frac{1}{2} \int_{\mathbb{R}_{+}^{N+1}}\left(\left|\left(-i \nabla_{x}-A_{\varepsilon}(x)\right) w\right|^{2}+\left|\frac{\partial w}{\partial y}\right|^{2}+m^{2} w^{2}\right) d x d y \\
& +\frac{1}{2} \int_{\mathbb{R}^{N}} V_{\varepsilon}(x)|y(w)|^{2} d x-\int_{\mathbb{R}^{N}} G_{\varepsilon}(x,|y(w)|) d x,
\end{aligned}
$$

which is $C^{1}$ with Gataux derivative

$$
\begin{aligned}
\left\langle\mathcal{J}_{\varepsilon}^{\prime}(w), v\right\rangle= & \Re \int_{\mathbb{R}_{+}^{N+1}} \nabla_{\tilde{A}_{\varepsilon}} w \overline{\nabla_{\tilde{A}_{\varepsilon}} v}+m^{2} w \bar{v} d x d y+\Re \int_{\mathbb{R}^{N}} V_{\varepsilon}(x) y(w) \overline{y(v)} d x \\
& -\Re \int_{\mathbb{R}^{N}} g_{\varepsilon}(x,|y(w)|) y(w) \overline{y(v)} d x,
\end{aligned}
$$

where $\nabla_{\tilde{A}_{\varepsilon}}$ is defined as (2.2).

Next, we define the Nehari manifold $\mathcal{N}_{\varepsilon}$ (see [35]) related to $\mathcal{J}_{\varepsilon}$. We say $w \in \mathcal{N}_{\varepsilon}$ means $w \in H_{A_{\varepsilon}}^{1}\left(\mathbb{R}_{+}^{N+1}, \mathbb{C}\right.$ ) and satisfies

$$
\begin{aligned}
& \int_{\mathbb{R}_{+}^{N+1}}\left(\left|\left(-i \nabla_{x}-A_{\varepsilon}(x)\right) w\right|^{2}+\left|\frac{\partial w}{\partial y}\right|^{2}+m^{2} w^{2}\right) d x d y+\int_{\mathbb{R}^{N}} V_{\varepsilon}(x)|y(w)|^{2} d x \\
& \quad=\int_{\mathbb{R}^{N}} g_{\varepsilon}(x,|y(w)|)|y(w)|^{2} d x .
\end{aligned}
$$

We denote by $\widetilde{H}_{A_{\varepsilon}}^{1}\left(\mathbb{R}_{+}^{N+1}, \mathbb{C}\right)$ the open subset of $H_{A_{\varepsilon}}^{1}\left(\mathbb{R}_{+}^{N+1}, \mathbb{C}\right)$ given by

$$
\widetilde{H}_{A_{\varepsilon}}^{1}\left(\mathbb{R}_{+}^{N+1}, \mathbb{C}\right)=\left\{w \in H_{A_{\varepsilon}}^{1}\left(\mathbb{R}_{+}^{N+1}, \mathbb{C}\right):|\operatorname{supp}| y(w)\left|\cap \mathcal{O}_{\varepsilon}\right|>0\right\},
$$

and $\widetilde{S}_{\varepsilon}=S_{\varepsilon} \cap \widetilde{H}_{A_{\varepsilon}}^{1}\left(\mathbb{R}_{+}^{N+1}, \mathbb{C}\right)$, where $S_{\varepsilon}$ is the unit sphere of $H_{A_{\varepsilon}}^{1}\left(\mathbb{R}_{+}^{N+1}, \mathbb{C}\right)$. Note that $\widetilde{S}_{\varepsilon}$ is a non-complete $C^{1,1}$-manifold of codimension 1 , modeled on $H_{A_{\varepsilon}}^{1}\left(\mathbb{R}_{+}^{N+1}, \mathbb{C}\right.$ ) and contained in the open $\widetilde{H}_{A_{\varepsilon}}^{1}\left(\mathbb{R}_{+}^{N+1}, \mathbb{C}\right.$ ) (see for example [32]). Then, $H_{A_{\varepsilon}}^{1}\left(\mathbb{R}_{+}^{N+1}, \mathbb{C}\right)=T_{w} \widetilde{S}_{\varepsilon} \bigoplus \mathbb{R} w$ for each $w \in \widetilde{S}_{\varepsilon}$, where

$$
T_{W} \widetilde{S}_{\varepsilon}=\left\{v \in H_{A_{\varepsilon}}^{1}\left(\mathbb{R}_{+}^{N+1}, \mathbb{C}\right):\langle w, v\rangle_{H_{A_{\varepsilon}}^{1}}=0\right\} .
$$

We can check the functional $\mathcal{J}_{\mathcal{\varepsilon}}$ satisfies the Mountain pass geometry [35].

Lemma 2.3. The functional $\mathcal{J}_{\varepsilon}$ satisfies the following conditions:

(i) There exist $\alpha, \rho>0$ such that $\mathcal{J}_{\varepsilon}(w) \geq \alpha$ with $\|w\|_{H_{A_{\varepsilon}}^{1}}=\rho$; 
(ii) There exists $e \in H_{A_{\varepsilon}}^{1}\left(\mathbb{R}_{+}^{N+1}, \mathbb{C}\right)$ such that $\|e\|_{H_{A_{\varepsilon}}^{1}}>\rho$ and $\mathcal{J}_{\mathcal{E}}(e)<0$.

Proof. (i) For any $w \in H_{A_{\varepsilon}}^{1}\left(\mathbb{R}_{+}^{N+1}, \mathbb{C}\right) \backslash\{0\}$ and $\varepsilon>0$ small, it follows from (g1)-(g2) that there exists $C_{\varepsilon}>0$ such that

$$
|g(\varepsilon x, s)| \leq \varepsilon+C_{\varepsilon} s^{2^{\sharp}-2} \text { for all } x \in \mathbb{R}^{N}, s \in \mathbb{R}
$$

and

$$
|G(\varepsilon x, s)| \leq \frac{\varepsilon}{2} s^{2}+\frac{C_{\varepsilon}}{2 \sharp} s^{2^{\sharp}} \quad \text { for all } x \in \mathbb{R}^{N}, s \in \mathbb{R} .
$$

Next, by the Sobolev embedding (2.4), (V1), (2.8) and (2.13), we have

$$
\begin{aligned}
\mathcal{J}_{\varepsilon}(w)= & \frac{1}{2} \int_{\mathbb{R}_{+}^{N+1}}\left(\left|\left(-i \nabla_{x}-A_{\varepsilon}(x)\right) w\right|^{2}+\left|\frac{\partial w}{\partial y}\right|^{2}+m^{2} w^{2}\right) d x d y \\
& +\frac{1}{2} \int_{\mathbb{R}^{N}}\left(V_{\varepsilon}(x)+V_{0}\right)|y(w)|^{2} d x-\frac{V_{0}}{2} \int_{\mathbb{R}^{N}}|y(w)|^{2} d x-\int_{\mathbb{R}^{N}} G_{\varepsilon}(x,|y(w)|) d x \\
\geq & \frac{1}{2} \int_{\mathbb{R}_{+}^{N+1}}\left(\left|\left(-i \nabla_{x}-A_{\varepsilon}(x)\right) w\right|^{2}+\left|\frac{\partial w}{\partial y}\right|^{2}+m^{2} w^{2}\right) d x d y \\
& -\frac{V_{0}}{2}\left(m \int_{\mathbb{R}_{+}^{N+1}}|w|^{2} d x d y+\frac{1}{m} \int_{\mathbb{R}_{+}^{N+1}}\left(\left|\left(-i \nabla_{x}-A_{\varepsilon}(x)\right) w\right|^{2}+\left|\frac{\partial w}{\partial y}\right|^{2}\right) d x d y\right) \\
& -\frac{\varepsilon}{2} \int_{\mathbb{R}^{N}}|y(w)|^{2} d x-\frac{C_{\varepsilon}}{2^{\sharp}} \int_{\mathbb{R}^{N}}|y(w)|^{2^{\sharp}} d x \\
\geq & \frac{1}{2} \\
& \left(1-\frac{V_{0}}{m}\right) \int_{\mathbb{R}_{+}^{N+1}}\left(\left|\left(-i \nabla_{x}-A_{\varepsilon}(x)\right) w\right|^{2}+\left|\frac{\partial w}{\partial y}\right|^{2}\right) d x d y \\
& +\frac{m\left(m-V_{0}\right)}{2} \int_{\mathbb{R}_{+}^{N+1}}|w|^{2} d x d y-\varepsilon C\|w\|_{H_{A_{\varepsilon}}^{1}}^{2}-C C_{\varepsilon}\|w\|_{H_{A_{\varepsilon}}^{\sharp}}^{2^{\sharp}}
\end{aligned}
$$

Therefore, we can choose $\alpha, \rho>0$ such that $\mathcal{J}_{\mathcal{E}}(w) \geq \alpha$ with $\|w\|_{H_{A_{\varepsilon}}^{1}}=\rho$ since $0<V_{0}<m$.

(ii) For every $w \in \widetilde{H}_{A_{\varepsilon}}^{1}\left(\mathbb{R}_{+}^{N+1}, \mathbb{C}\right)$ and $t>0$, we can obtain that

$$
\begin{aligned}
\mathcal{J}_{\varepsilon}(t w) \leq & \frac{t^{2}}{2} \int_{\mathbb{R}_{+}^{N+1}}\left(\left|\left(-i \nabla_{x}-A_{\varepsilon}(x)\right) w\right|^{2}+\left|\frac{\partial w}{\partial y}\right|^{2}+m^{2} w^{2}\right) d x d y+\frac{t^{2}}{2} \int_{\mathbb{R}^{N}} V_{\varepsilon}(x)|y(w)|^{2} d x \\
& -\int_{\mathcal{O}_{\varepsilon}} G_{\varepsilon}(x, t|y(w)|) d x \\
\leq & \frac{t^{2}}{2} \int_{\mathbb{R}_{+}^{N+1}}\left(\left|\left(-i \nabla_{x}-A_{\varepsilon}(x)\right) w\right|^{2}+\left|\frac{\partial w}{\partial y}\right|^{2}+m^{2} w^{2}\right) d x d y+\frac{t^{2}}{2} \int_{\mathbb{R}^{N}} V_{\varepsilon}(x)|y(w)|^{2} d x \\
& \quad-C_{1} t^{\theta} \int_{\mathcal{O}_{\varepsilon}}|y(w)|^{\theta} d x+C_{2}|\operatorname{supp}| y(w)\left|\cap \mathcal{O}_{\varepsilon}\right|,
\end{aligned}
$$

where we have used (g3) and the standard ODE computations. This and (2.4) imply the conclusion (ii) since $\theta \in\left(2,2^{\sharp}\right)$.

Since we only assume $f$ is continuous, in order to overcome the non-differential of $\mathcal{N}_{\varepsilon}$ and the in completeness of $\widetilde{S}_{\varepsilon}$, we need the following two results.

Lemma 2.4. Assume that assumptions $(A),(V 1)-(V 2)$ and $(f 1)-(f 4)$ are satisfied. Then the following properties hold: 
(a) For each $w \in \widetilde{H}_{A_{\varepsilon}}^{1}\left(\mathbb{R}_{+}^{N+1}, \mathbb{C}\right)$, let $h_{w}: \mathbb{R}^{+} \rightarrow \mathbb{R}$ be defined as $h_{w}(s)=\mathcal{J}_{\varepsilon}(s w)$. Then there exists a unique $s_{w}>0$ such that $h_{w}^{\prime}(s)>0$ in $\left(0, s_{w}\right)$ and $h_{w}^{\prime}(s)<0$ in $\left(s_{w}, \infty\right)$.

(b) There is a $t>0$ independent of $w$ such that $s_{w}>t$ for all $w \in \widetilde{S}_{\varepsilon}$. Moreover, for each compact set $\mathcal{W} \subset \widetilde{S}_{\varepsilon}$, there is $C_{\mathcal{W}}>0$ such that $s_{w} \leq C_{\mathcal{W}}$ for all $w \in \mathcal{W}$.

(c) The map $\widehat{m}_{\varepsilon}: \widetilde{S}_{\varepsilon} \rightarrow \mathcal{N}_{\varepsilon}$ given by $\widehat{m}_{\varepsilon}(w)=s_{w} w$ is continuous and $m_{\varepsilon}:=\left.\widehat{m}_{\varepsilon}\right|_{\widetilde{S}_{\varepsilon}}$ is a homeomorphism between $\widetilde{S}_{\varepsilon}$ and $\mathcal{N}_{\varepsilon}$. Moreover, $m_{\varepsilon}^{-1}(w)=\frac{w}{\|w\|_{H_{A_{\varepsilon}}^{1}}}$.

(d) If there is a sequence $\left\{w_{n}\right\} \subset \widetilde{S}_{\varepsilon}$ such that dist $\left(w_{n}, \partial \widetilde{S}_{\varepsilon}\right) \rightarrow 0$, then $\left\|m_{\varepsilon}\left(w_{n}\right)\right\|_{H_{A_{\varepsilon}}^{1}} \rightarrow \infty$ and $\mathcal{J}_{\varepsilon}\left(m_{\varepsilon}\left(w_{n}\right)\right) \rightarrow$ $\infty$.

Proof. (a) Observe from the proof of Lemma 2.3 that $h_{w}(0)=0, h_{w}(s)>0$ for $s$ small and $h_{w}(s)<0$ for $s$ large. Thus $\max _{s \geq 0} h_{w}(s)$ is achieved at a $s=s_{w}>0$ satisfying $h_{w}^{\prime}\left(s_{w}\right)=0$ and $s_{w} w \in \mathcal{N}_{\varepsilon}$. On the other hand, we know

$$
\begin{aligned}
h_{w}^{\prime}(s)=0 \Leftrightarrow s w & \in \mathcal{N}_{\varepsilon} \\
& \Leftrightarrow \int_{\mathbb{R}_{+}^{N+1}}\left(\left|\left(-i \nabla_{x}-A_{\varepsilon}(x)\right) w\right|^{2}+\left|\frac{\partial w}{\partial y}\right|^{2}+m^{2} w^{2}\right) d x d y+\int_{\mathbb{R}^{N}} V_{\varepsilon}(x)|y(w)|^{2} d x \\
& =\int_{\mathbb{R}^{N}} g_{\varepsilon}(x, s|y(w)|)|y(w)|^{2} d x .
\end{aligned}
$$

By the definition of $g$, the right hand side is nondecreasing in $s$ for $s>0$. Therefore, $\max _{s \geq 0} h_{w}(s)$ is achieved at a unique $s=s_{w}>0$ such that $h_{w}^{\prime}\left(s_{w}\right)=0$ and $s_{w} w \in \mathcal{N}_{\varepsilon}$.

(b) Assume that $w \in \widetilde{S}_{\varepsilon}$, then by (2.15), (2.8), (2.12) and a similar argument as (2.14), we can get that $s_{w} \geq t$ for some $t>0$. Suppose by contradiction that there is $\left\{w_{n}\right\} \subset \mathcal{W}$ with $s_{n}:=s_{w_{n}} \rightarrow \infty$. Since $\mathcal{W}$ is compact, there exists a $w \in \mathcal{W}$ such that $w_{n} \rightarrow w$ in $\widetilde{H}_{A_{\varepsilon}}^{1}\left(\mathbb{R}_{+}^{N+1}, \mathbb{C}\right)$. Moreover $\mathcal{J}_{\varepsilon}\left(s_{n} w_{n}\right) \rightarrow-\infty$.

On the other hand, denote $v_{n}=s_{n} w_{n} \in \mathcal{N}_{\varepsilon}$ and use (2.8) and property (g3), we have that

$$
\begin{aligned}
\mathcal{J}_{\mathcal{E}}\left(v_{n}\right)= & \mathcal{J}_{\varepsilon}\left(v_{n}\right)-\frac{1}{\mu}\left\langle\mathcal{J}_{\varepsilon}^{\prime}\left(v_{n}\right), v_{n}\right\rangle \\
= & \left(\frac{1}{2}-\frac{1}{\mu}\right)\left(\int_{\mathbb{R}_{+}^{N+1}}\left(\left|\left(-i \nabla_{x}-A_{\varepsilon}(x)\right) v_{n}\right|^{2}+\left|\frac{\partial v_{n}}{\partial y}\right|^{2}+m^{2} v_{n}^{2}\right) d x d y\right. \\
& \left.+\int_{\mathbb{R}^{N}} V_{\varepsilon}(x)\left|y\left(v_{n}\right)\right|^{2} d x\right)+\frac{1}{\mu} \int_{\mathbb{R}^{N}}\left(g_{\varepsilon}\left(x,\left|y\left(v_{n}\right)\right|\right)\left|y\left(v_{n}\right)\right|^{2}-\mu G_{\varepsilon}\left(x,\left|y\left(v_{n}\right)\right|\right)\right) d x \\
\geq & \left(\frac{1}{2}-\frac{1}{\mu}\right)\left(\int_{\mathbb{R}_{+}^{N+1}}\left(\left|\left(-i \nabla_{x}-A_{\varepsilon}(x)\right) v_{n}\right|^{2}+\left|\frac{\partial v_{n}}{\partial y}\right|^{2}+m^{2} v_{n}^{2}\right) d x d y\right. \\
& \left.+\int_{\mathbb{R}^{N}} V_{\varepsilon}(x)\left|y\left(v_{n}\right)\right|^{2} d x\right)-\left(\frac{1}{2}-\frac{1}{\mu}\right) \int_{\mathbb{R}^{N}} \frac{V_{0}\left|y\left(v_{n}\right)\right|^{2}}{K} d x \\
= & \left(\frac{1}{2}-\frac{1}{\mu}\right)\left(\int_{\mathbb{R}_{+}^{N+1}}\left(\left|\left(-i \nabla_{x}-A_{\varepsilon}(x)\right) v_{n}\right|^{2}+\left|\frac{\partial v_{n}}{\partial y}\right|^{2}+m^{2} v_{n}^{2}\right) d x d y\right) \\
& +\left(\frac{1}{2}-\frac{1}{\mu}\right)\left(\int_{\mathbb{R}^{N}}\left(V_{\varepsilon}(x)+V_{0}\right)\left|y\left(v_{n}\right)\right|^{2} d x-\left(1+\frac{1}{K}\right) V_{0} \int\left|y\left(v_{n}\right)\right|^{2} d x\right) \\
\geq & \left(\frac{1}{2}-\frac{1}{\mu}\right)\left(\int_{\mathbb{R}_{+}^{N+1}}\left(\left|\left(-i \nabla_{x}-A_{\varepsilon}(x)\right) v_{n}\right|^{2}+\left|\frac{\partial v_{n}}{\partial y}\right|^{2}+m^{2} v_{n}^{2}\right) d x d y\right)
\end{aligned}
$$




$$
\begin{aligned}
& -\left(\frac{1}{2}-\frac{1}{\mu}\right)\left(1+\frac{1}{K}\right) V_{0} \int_{\mathbb{R}^{N}}\left|y\left(v_{n}\right)\right|^{2} d x \\
\geq( & \left.\frac{1}{2}-\frac{1}{\mu}\right)\left(\int_{\mathbb{R}_{+}^{N+1}}\left(\left|\left(-i \nabla_{x}-A_{\varepsilon}(x)\right) v_{n}\right|^{2}+\left|\frac{\partial v_{n}}{\partial y}\right|^{2}+m^{2} v_{n}^{2}\right) d x d y\right) \\
& -\left(\frac{1}{2}-\frac{1}{\mu}\right)\left(1+\frac{1}{K}\right) V_{0}\left(\frac{1}{m} \int_{\mathbb{R}_{+}^{N+1}}\left(\left|\left(-i \nabla_{x}-A_{\varepsilon}(x)\right) w\right|^{2}+\left|\frac{\partial w}{\partial y}\right|^{2}\right) d x d y\right. \\
& \left.+m \int_{\mathbb{R}_{+}^{N+1}}|w|^{2} d x d y\right) \\
=( & \left.\frac{1}{2}-\frac{1}{\mu}\right)\left(1-\left(1+\frac{1}{K}\right) \frac{V_{0}}{m}\right) \int_{\mathbb{R}_{+}^{N+1}}\left(\left|\left(-i \nabla_{x}-A_{\varepsilon}(x)\right)^{2} w\right|^{2}+\left|\frac{\partial w}{\partial y}\right|^{2}\right) d x d y \\
& +\left(\frac{1}{2}-\frac{1}{\mu}\right) m\left(m-\left(1+\frac{1}{K}\right) V_{0}\right) \int_{\mathbb{R}_{+}^{N+1}}|w|^{2} d x d y
\end{aligned}
$$

Therefore, we can prove $\mathcal{J}_{\varepsilon}\left(v_{n}\right)>0$ since $K>\frac{V_{0}}{m-V_{0}}$ and $\mu>2$, which yields a contradiction.

(c) We first show that $\widehat{m}_{\varepsilon}, m_{\varepsilon}$ and $m_{\varepsilon}^{-1}$ are well defined. In fact, by (a), we know that for each $w \in$ $\widetilde{H}_{A_{\varepsilon}}^{1}\left(\mathbb{R}_{+}^{N+1}, \mathbb{C}\right)$, there is a unique $\widehat{m}_{\varepsilon}(w) \in \mathcal{N}_{\varepsilon}$. On the other hand, we claim that if $w \in \mathcal{N}_{\varepsilon}$, then $w \in$ $\widetilde{H}_{A_{\varepsilon}}^{1}\left(\mathbb{R}_{+}^{N+1}, \mathbb{C}\right)$. Otherwise, we have $|\operatorname{supp}| y(w)\left|\cap \mathcal{O}_{\varepsilon}\right|=0$ and by (2.8) and (g3)-(ii), we have

$$
\begin{aligned}
\int_{\mathbb{R}_{+}^{N+1}} & \left(\left|\left(-i \nabla_{x}-A_{\varepsilon}(x)\right) w\right|^{2}+\left|\frac{\partial w}{\partial y}\right|^{2}+m^{2} w^{2}\right) d x d y \\
& =-\int_{\mathbb{R}^{N}} V_{\varepsilon}(x)|y(w)|^{2} d x+\int_{\mathbb{R}^{N}} g_{\varepsilon}(x,|y(w)|)|y(w)|^{2} d x \\
& =-\int_{\mathbb{R}^{N}} V_{\varepsilon}(x)|y(w)|^{2} d x+\int_{\mathbb{R}^{N} \backslash \mathcal{O}_{\varepsilon}} g_{\varepsilon}(x,|y(w)|)|y(w)|^{2} d x \\
& \leq-\int_{\mathbb{R}^{N}} V_{\varepsilon}(x)|y(w)|^{2} d x+\frac{V_{0}}{K} \int_{\mathbb{R}^{N} \mid \mathcal{O}_{\varepsilon}}|y(w)|^{2} d x \\
& =-\int_{\mathbb{R}^{N}}\left(V_{\varepsilon}(x)+V_{0}\right)|y(w)|^{2} d x+\left(1+\frac{1}{K}\right) V_{0} \int_{\mathbb{R}^{N}}|y(w)|^{2} d x \\
& \leq\left(1+\frac{1}{K}\right) V_{0} \int_{\mathbb{R}^{N}}|y(w)|^{2} d x \\
& \leq\left(1+\frac{1}{K}\right) V_{0}\left(m \int_{\mathbb{R}_{+}^{N+1}}|w|^{2} d x d y+\frac{1}{m} \int_{\mathbb{R}_{+}^{N+1}}\left(\left|\left(-i \nabla_{x}-A_{\varepsilon}(x)\right) w\right|^{2}+\left|\frac{\partial w}{\partial y}\right|^{2}\right) d x d y\right) .
\end{aligned}
$$

This implies that

$$
\begin{aligned}
(1- & \left.\left(1+\frac{1}{K}\right) \frac{V_{0}}{m}\right) \int_{\mathbb{R}_{+}^{N+1}}\left(\left|\left(-i \nabla_{x}-A_{\varepsilon}(x)\right) w\right|^{2}+\left|\frac{\partial w}{\partial y}\right|^{2}\right) d x d y \\
& +m\left(m-\left(1+\frac{1}{K}\right) V_{0}\right) \int_{\mathbb{R}_{+}^{N+1}}|w|^{2} d x d y
\end{aligned}
$$

$\leq 0$, 
which is impossible since $K>\frac{V_{0}}{m-V_{0}}$ and $w \not \equiv 0$. Therefore, $m_{\varepsilon}^{-1}(w)=\frac{w}{\|w\|_{H_{A_{\varepsilon}}^{1}}} \in \widetilde{S}_{\varepsilon}$ is well defined and continuous. Since

$$
m_{\varepsilon}^{-1}\left(m_{\varepsilon}(w)\right)=m_{\varepsilon}^{-1}\left(s_{w} w\right)=\frac{s_{w} w}{s_{w}\|w\|_{H_{A_{\varepsilon}}^{1}}}=w, \quad \text { for } w \in \widetilde{S}_{\varepsilon},
$$

we conclude that $m_{\varepsilon}$ is a bijection. Next, we prove $\widehat{m}_{\varepsilon}: \widetilde{H}_{A_{\varepsilon}}^{1}\left(\mathbb{R}_{+}^{N+1}, \mathbb{C}\right) \rightarrow \mathcal{N}_{\varepsilon}$ is continuous. Let $\left\{w_{n}\right\} \subset$ $\widetilde{H}_{A_{\varepsilon}}^{1}\left(\mathbb{R}_{+}^{N+1}, \mathbb{C}\right)$ and $w \in \widetilde{H}_{A_{\varepsilon}}^{1}\left(\mathbb{R}_{+}^{N+1}, \mathbb{C}\right)$ such that $w_{n} \rightarrow w$ in $H_{A_{\varepsilon}}^{1}\left(\mathbb{R}_{+}^{N+1}, \mathbb{C}\right)$. By (b), there is a $s_{0}>0$ such that $s_{n}:=s_{w_{n}} \rightarrow s_{0}$. Since $s_{n} w_{n} \in \mathcal{N}_{\varepsilon}$, we have

$$
\begin{aligned}
\int_{\mathbb{R}_{+}^{N+1}} & \left(\left|\left(-i \nabla_{x}-A_{\varepsilon}(x)\right) w_{n}\right|^{2}+\left|\frac{\partial w_{n}}{\partial y}\right|^{2}+m^{2} w_{n}^{2}\right) d x d y+\int_{\mathbb{R}^{N}} V_{\varepsilon}(x)\left|y\left(w_{n}\right)\right|^{2} d x \\
& =\int_{\mathbb{R}^{N}} g_{\varepsilon}\left(x, s_{n}\left|y\left(w_{n}\right)\right|\right)\left|y\left(w_{n}\right)\right|^{2} d x .
\end{aligned}
$$

Then, by passing to the limit as $n \rightarrow+\infty$, we have

$$
\begin{aligned}
& \int_{\mathbb{R}_{+}^{N+1}}\left(\left|\left(-i \nabla_{x}-A_{\varepsilon}(x)\right) w\right|^{2}+\left|\frac{\partial w}{\partial y}\right|^{2}+m^{2} w^{2}\right) d x d y+\int_{\mathbb{R}^{N}} V_{\varepsilon}(x)|y(w)|^{2} d x \\
& \quad=\int_{\mathbb{R}^{N}} g_{\varepsilon}\left(x, s_{0}|y(w)|\right)|y(w)|^{2} d x,
\end{aligned}
$$

which means that $s_{0} w \in \mathcal{N}_{\varepsilon}$ and $s_{w}=s_{0}$. This implies $\widehat{m}_{\varepsilon}\left(w_{n}\right) \rightarrow \widehat{m}_{\varepsilon}(w)$ in $\widetilde{H}_{A_{\varepsilon}}^{1}\left(\mathbb{R}_{+}^{N+1}, \mathbb{C}\right)$. So $\widehat{m}_{\varepsilon}$ and $m_{\varepsilon}$ are continuous functions.

(d) Let $\left\{w_{n}\right\} \subset \widetilde{S}_{\varepsilon}$ be a sequence such that $\operatorname{dist}\left(w_{n}, \partial \widetilde{S}_{\varepsilon}\right) \rightarrow 0$. Therefore, for each $v \in \partial \widetilde{S}_{\varepsilon}$ and $n \in \mathbb{N}$, we have

$$
\left|y\left(w_{n}\right)\right| \leq\left|y\left(w_{n}\right)-y(v)\right| \text { a.e. in } \mathcal{O}_{\varepsilon} .
$$

Then by the Sobolev embedding, there exits constant $C_{t}>0$ such that

$$
\begin{aligned}
& \left|y\left(w_{n}\right)\right|_{L^{t}\left(\mathcal{O}_{\varepsilon}\right)} \\
& \quad \leq \inf _{v \in \partial \widetilde{S}_{\varepsilon}}\left|y\left(w_{n}\right)-y(v)\right|_{L^{t}\left(\mathcal{O}_{\varepsilon}\right)} \\
& \quad \leq C_{t} \inf _{v \in \partial \widetilde{S}_{\varepsilon}}\left(\int_{\mathbb{R}_{+}^{N+1}}\left(\left|\left(-i \nabla_{x}-A_{\varepsilon}(x)\right)\left(w_{n}-v\right)\right|^{2}+\left|\frac{\partial\left(w_{n}-v\right)}{\partial y}\right|^{2}+\left(w_{n}-v\right)^{2}\right) d x d y\right) \\
& \quad=C_{t} \operatorname{dist}\left(w_{n}, \partial \widetilde{S}_{\varepsilon}\right)
\end{aligned}
$$

for all $m \in \mathbb{N}$ and $t \in\left[2,2^{\sharp}\right]$. By (g1)-(g2), (g3)-(ii) and (2.19), for $s>0$, we have

$$
\begin{aligned}
\int_{\mathbb{R}^{N}} G_{\varepsilon}\left(x, s w_{n}\right) d x \leq & C_{1} s^{2} \int_{\mathcal{O}_{\varepsilon}}\left|y\left(w_{n}\right)\right|^{2} d x+C_{2} s^{2^{\sharp}} \int_{\mathcal{O}_{\varepsilon}}\left|y\left(w_{n}\right)\right|^{2^{\sharp}} d x \\
& +s^{2} \frac{V_{0}}{K} \int_{\mathbb{R}^{N} \backslash \mathcal{O}_{\varepsilon}}\left|y\left(w_{n}\right)\right|^{2} d x \\
\leq & C_{3} s^{2} \operatorname{dist}\left(w_{n}, \partial \widetilde{S}_{\varepsilon}\right)^{2}+C_{4} s^{2^{\sharp}} \operatorname{dist}\left(w_{n}, \partial \widetilde{S}_{\varepsilon}\right)^{2^{\sharp}} \\
& +s^{2} \frac{V_{0}}{K} \int_{\mathbb{R}^{N} \backslash \mathcal{O}_{\varepsilon}}\left|y\left(w_{n}\right)\right|^{2} d x .
\end{aligned}
$$

On the other hand, from the definition of $m_{\varepsilon}$, for all $s>0$, we have

$$
\liminf _{n \rightarrow \infty} \mathcal{J}_{\varepsilon}\left(m_{\varepsilon}\left(w_{n}\right)\right)
$$




$$
\begin{aligned}
\geq & \liminf _{n \rightarrow \infty} \mathcal{J}_{\varepsilon}\left(s w_{n}\right) \\
\geq & \liminf _{n \rightarrow \infty} \frac{s^{2}}{2}\left(\int_{\mathbb{R}_{+}^{N+1}}\left(\left|\left(-i \nabla_{x}-A_{\varepsilon}(x)\right) w\right|^{2}+\left|\frac{\partial w}{\partial y}\right|^{2}+m^{2} w^{2}\right) d x d y\right. \\
& \left.+\int_{\mathbb{R}^{N}}\left(V_{\varepsilon}(x)+V_{0}\right)|y(w)|^{2} d x\right)-\limsup _{n \rightarrow \infty}\left(s^{2} V_{0}\left(\frac{1}{2}+\frac{1}{K}\right) \int_{\mathbb{R}^{N} \backslash \mathcal{O}_{\varepsilon}}\left|y\left(w_{n}\right)\right|^{2} d x\right. \\
& \left.+C_{3} s^{2} \operatorname{dist}\left(w_{n}, \partial \widetilde{S}_{\varepsilon}\right)^{2}+C_{4} s^{2^{\sharp}} \operatorname{dist}\left(w_{n}, \partial \widetilde{S}_{\varepsilon}\right)^{2^{\sharp}}\right) \\
\geq & \liminf _{n \rightarrow \infty} s^{2}\left(\frac{1}{2}-\left(\frac{1}{2}+\frac{1}{K}\right) \frac{V_{0}}{m}\right) \int_{\mathbb{R}_{+}^{N+1}}\left(\left|\left(-i \nabla_{x}-A_{\varepsilon}(x)\right) w\right|^{2}+\left|\frac{\partial w}{\partial y}\right|^{2}+m^{2} w^{2}\right) d x d y \\
& +\liminf _{n \rightarrow \infty} s^{2}\left(\frac{m^{2}}{2}-\left(\frac{1}{2}+\frac{1}{K}\right) m V_{0}\right) \int_{\mathbb{R}_{+}^{N+1}} w^{2} d x d y \\
& -\limsup _{n \rightarrow \infty}\left(C_{3} s^{2} \operatorname{dist}\left(w_{n}, \partial \widetilde{S}_{\varepsilon}\right)^{2}+C_{4} s^{2^{\sharp}} \operatorname{dist}\left(w_{n}, \partial \widetilde{S}_{\varepsilon}\right)^{2^{\sharp}}\right),
\end{aligned}
$$

where we have used inequality (2.8). Thus, by the arbitrary of $s>0$, we conclude $\mathcal{J}_{\mathcal{E}}\left(m_{\varepsilon}\left(w_{n}\right)\right) \rightarrow \infty$ by (2.20). Similarly, we can get $\left\|m_{\varepsilon}\left(w_{n}\right)\right\|_{H_{A_{\varepsilon}}^{1}} \rightarrow \infty$ from (2.8).

Now we define the function

$$
\widehat{\Phi}_{\varepsilon}: \widetilde{H}_{A_{\varepsilon}}^{1}\left(\mathbb{R}_{+}^{N+1}, \mathbb{C}\right) \rightarrow \mathbb{R}
$$

as

$$
\widehat{\Phi}_{\varepsilon}(w)=\mathcal{J}_{\varepsilon}\left(\widehat{m}_{\varepsilon}(w)\right)
$$

and denote by $\Phi_{\varepsilon}:=\left.\widehat{\Phi}_{\varepsilon}\right|_{\widetilde{S}_{\varepsilon}}$. A direct conclusion of Lemma 2.4 is the following.

Proposition 2.5. Assume that assumptions $(A),(V 1)-(V 2)$ and $(f 1)-(f 4)$ are satisfied. Then

(a) $\widehat{\Phi}_{\varepsilon} \in C^{1}\left(\widetilde{H}_{A_{\varepsilon}}^{1}, \mathbb{R}\right)$ and $\Phi_{\varepsilon} \in C^{1}\left(\widetilde{S}_{\varepsilon}, \mathbb{R}\right)$.

(b) If $\left\{w_{n}\right\}$ is a $(P S)_{c}$ sequence of $\Phi_{\varepsilon}$, then $\left\{m_{\varepsilon}\left(w_{n}\right)\right\}$ is a $(P S)_{c}$ sequence of $\mathcal{J}_{\varepsilon}$. If $\left\{w_{n}\right\} \subset \mathcal{N}_{\varepsilon}$ is a bounded $(P S)_{c}$ sequence of $\mathcal{J}_{\varepsilon}$, then $\left\{m_{\varepsilon}^{-1}\left(w_{n}\right)\right\}$ is a $(P S)_{c}$ sequence of $\Phi_{\varepsilon}$.

(c) $w$ is a critical point of $\Phi_{\varepsilon}$ if and only if $m_{\varepsilon}(w)$ is a critical point of $\mathcal{J}_{\mathcal{\varepsilon}}$. Moreover, we have

$$
\inf _{\widetilde{S}_{\varepsilon}} \Phi_{\varepsilon}=\inf _{\mathcal{N}_{\varepsilon}} \mathcal{J}_{\varepsilon} .
$$

Proof. The details of the proof can be found in relevant material from Corollary 2.3 in [32], and we omit it here.

As in [32], we have the following variational characterization of the infimum of $\mathcal{J}_{\varepsilon}$ under $\mathcal{N}_{\varepsilon}$ :

$$
c_{\varepsilon}=\inf _{w \in \mathcal{N}_{\varepsilon}} \mathcal{J}_{\varepsilon}=\inf _{w \in \widetilde{H}_{A_{\varepsilon}}^{1}} \max _{t>0} \mathcal{J}_{\varepsilon}(t w)=\inf _{w \in \widetilde{S}_{\varepsilon}} \max _{t>0} \mathcal{J}_{\varepsilon}(t w)>0 .
$$

The main feature of the modified functional is that it satisfies the local compactness condition, we will show it as follows.

Lemma 2.6. Let $c>0$ and $\left\{w_{n}\right\}$ be $a(P S)_{c}$ sequence for $\mathcal{J}_{\varepsilon}$, then $\left\{w_{n}\right\}$ is bounded $H_{A_{\varepsilon}}^{1}\left(\mathbb{R}_{+}^{N+1}, \mathbb{C}\right)$.

Proof. Suppose $\left\{w_{n}\right\}$ is a $(P S)_{c}$ sequence for $\mathcal{J}_{\mathcal{E}}$, then $\mathcal{J}_{\mathcal{E}}\left(w_{n}\right)=c+o_{n}(1)$ and $\mathcal{J}_{\varepsilon}^{\prime}\left(w_{n}\right)=o_{n}(1)$, where $o_{n}(1) \rightarrow 0$ as $n \rightarrow \infty$. Then, by a similar argument of (2.16), we can get the conclusion. 
Lemma 2.7. Let $c>0$ and $\left\{w_{n}\right\}$ be $a(P S)_{c}$ sequence for $\mathcal{J}_{\mathcal{E}}$, then for each $\zeta>0$, there is a number $R=R(\zeta)>0$ such that

$$
\limsup _{n \rightarrow \infty} \int_{\mathbb{R}_{+}^{N+1} \backslash B_{R}^{+}}\left|\nabla_{\tilde{A}_{\varepsilon}} w_{n}\right|^{2}+m^{2} w_{n}^{2} d x d y+\int_{\mathbb{R}^{N} \backslash B_{R}}\left(V_{\varepsilon}(x)+V_{0}\right)\left|y\left(w_{n}\right)\right|^{2} d x<\zeta,
$$

where $(x, y) \in B_{R}^{+}:=B_{R} \times\{y>0\}$.

Proof. For $R>0$, let $\eta_{R} \in C^{\infty}\left(\mathbb{R}_{+}^{N+1}\right)$ be such that $\eta_{R}(x, y)=0$ if $(x, y) \in B_{R / 2}^{+}$and $\eta_{R}(x, y)=1$ if $(x, y) \notin B_{R}^{+}$, with $0 \leq \eta_{R}(x, y) \leq 1,\left|\nabla \eta_{R}\right| \leq C / R$ where $C$ is constant independent on $R$. Since $\left\{\eta_{R} w_{n}\right\}$ is bounded in $H_{A_{\varepsilon}}^{1}\left(\mathbb{R}_{+}^{N+1}, \mathbb{C}\right)$, it follows that $\left\langle J_{\varepsilon}^{\prime}\left(w_{n}\right), \eta_{R} w_{n}\right\rangle=o_{n}(1)$. Therefore,

$$
\begin{aligned}
& \Re \int_{\mathbb{R}_{+}^{N+1}} \nabla_{\tilde{A}_{\varepsilon}} w_{n} \overline{\nabla_{\tilde{A}_{\varepsilon}} \eta_{R} w_{n}}+m^{2} w_{n} \overline{\eta_{R} w_{n}} d x d y+\int_{\mathbb{R}^{N}} V_{\varepsilon}(x)\left|y\left(w_{n}\right)\right|^{2} \eta_{R} d x \\
& =\int_{\mathbb{R}^{N}} g_{\varepsilon}\left(x,\left|y\left(w_{n}\right)\right|\right)\left|y\left(w_{n}\right)\right|^{2} \eta_{R} d x+o_{n}(1) .
\end{aligned}
$$

Since $\overline{\nabla_{\tilde{A}_{\varepsilon}} \eta_{R} w_{n}}=i \overline{w_{n}} \nabla \eta_{R}+\eta_{R} \overline{\nabla_{\tilde{A}_{\varepsilon}} w_{n}}$, then we have

$$
\begin{aligned}
& \int_{\mathbb{R}_{+}^{N+1}} \eta_{R}\left(\left|\nabla_{\tilde{A}_{\varepsilon}} w_{n}\right|^{2}+m^{2}\left|w_{n}\right|^{2}\right) d x d y+\int_{\mathbb{R}^{N}} \eta_{R} V_{\varepsilon}(x)\left|y\left(w_{n}\right)\right|^{2} d x \\
& =\int_{\mathbb{R}^{N}} g_{\varepsilon}\left(x,\left|y\left(w_{n}\right)\right|\right)\left|y\left(w_{n}\right)\right|^{2} \eta_{R} d x-\Re \int_{B_{R}^{+} \mid B_{R / 2}^{+}} i \overline{w_{n}} \nabla \eta_{R} \nabla_{\tilde{A}_{\varepsilon}} w_{n} d x d y+o_{n}(1) .
\end{aligned}
$$

Then, fixing $R>0$ such that $\mathcal{O}_{\varepsilon} \subset B_{R / 2}$ and using (g3)-(ii) and Hölder inequality, we can get

$$
\begin{aligned}
& \int_{\mathbb{R}_{+}^{N+1}} \eta_{R}\left(\left|\nabla_{\tilde{A}_{\varepsilon}} w_{n}\right|^{2}+m^{2}\left|w_{n}\right|^{2}\right) d x d y+\int_{\mathbb{R}^{N}} \eta_{R} V_{\varepsilon}(x)\left|y\left(w_{n}\right)\right|^{2} d x \\
& \leq \frac{V_{0}}{K} \int_{\mathbb{R}^{N}}\left|y\left(w_{n}\right)\right|^{2} \eta_{R} d x+\frac{C}{R}\left(\int_{B_{R}^{+} \backslash B_{R / 2}^{+}}\left|\nabla_{\tilde{A}_{\varepsilon}} w_{n}\right|^{2} d x d y\right)^{\frac{1}{2}}\left(\int_{B_{R}^{+} \backslash B_{R / 2}^{+}}\left|w_{n}\right|^{2} d x d y\right)^{\frac{1}{2}}+o_{n}(1)
\end{aligned}
$$

By (2.8), we have

$$
\begin{aligned}
& \int_{\mathbb{R}_{+}^{N+1}} \eta_{R}\left(\left|\nabla_{\tilde{A}_{\varepsilon}} w_{n}\right|^{2}+m^{2}\left|w_{n}\right|^{2}\right) d x d y+\int_{\mathbb{R}^{N}} \eta_{R}\left(V_{\varepsilon}+V_{0}\right)(x)\left|y\left(w_{n}\right)\right|^{2} d x \\
& \quad \leq\left(\min \left\{\left(1-\left(1+\frac{1}{K}\right) \frac{V_{0}}{m}\right), m\left(m-\left(1+\frac{1}{K}\right) V_{0}\right)\right\}\right)^{-1}\left(\frac{C}{R}\left\|w_{n}\right\|_{H_{A_{\varepsilon}}^{1}}^{2}+o_{n}(1)\right) .
\end{aligned}
$$

We get the conclusion by choosing $R$ large, using he boundedness of $\left\{w_{n}\right\}$ proved in Lemma 2.6 and passing to the limit in the last inequality.

Lemma 2.8. The functional $\mathcal{J}_{\mathcal{E}}$ satisfies the Palais-Smale condition for any level $c \in \mathbb{R}$.

Proof. By Lemma 2.6, we know $\left\{w_{n}\right\}$ is bounded in $H_{A_{\varepsilon}}^{1}\left(\mathbb{R}_{+}^{N+1}, \mathbb{C}\right)$ and thus we can suppose that $w_{n} \rightarrow w$ weakly in $H_{A_{\varepsilon}}^{1}\left(\mathbb{R}_{+}^{N+1}, \mathbb{C}\right)$. In view of $\mathcal{J}_{\varepsilon}^{\prime}\left(w_{n}\right) \rightarrow 0$, the local compactness of $H_{A_{\varepsilon}}^{1}\left(\mathbb{R}_{+}^{N+1}, \mathbb{C}\right)$ and the subcritical growth of $g$, one has $\mathcal{J}_{\varepsilon}^{\prime}(w)=0$, that is

$$
\int_{\mathbb{R}_{+}^{N+1}}\left(\left|\nabla_{\tilde{A}_{\varepsilon}} w\right|^{2}+m^{2}|w|^{2}\right) d x d y+\int_{\mathbb{R}^{N}} V_{\varepsilon}(x)|y(w)|^{2} d x=\int_{\mathbb{R}^{N}} g_{\varepsilon}(x,|y(w)|)|y(w)|^{2} d x .
$$


On the other hand, using Lemma 2.7, we can prove that

$$
\begin{aligned}
& \int_{\mathbb{R}^{N}} g_{\varepsilon}\left(x,\left|y\left(w_{n}\right)\right|\right)\left|y\left(w_{n}\right)\right|^{2} d x \rightarrow \int_{\mathbb{R}^{N}} g_{\varepsilon}(x,|y(w)|)|y(w)|^{2} d x, \\
& \int_{\mathbb{R}^{N}}\left(V_{\varepsilon}(x)+V_{0}\right)\left|y\left(w_{n}\right)\right|^{2} d x \rightarrow \int_{\mathbb{R}^{N}}\left(V_{\varepsilon}(x)+V_{0}\right)|y(w)|^{2} d x, \\
& \int_{\mathbb{R}^{N}} V_{0}\left|y\left(w_{n}\right)\right|^{2} d x \rightarrow \int_{\mathbb{R}^{N}} V_{0}|y(w)|^{2} d x,
\end{aligned}
$$

as $n \rightarrow \infty$. Combining (2.21)-(2.24) and $\mathcal{J}_{\varepsilon}^{\prime}\left(w_{n}\right) \rightarrow 0$, we have $w_{n} \rightarrow w$ strongly in $H_{A_{\varepsilon}}^{1}\left(\mathbb{R}_{+}^{N+1}, \mathbb{C}\right)$.

We finish this section by showing $\Phi_{\varepsilon}$ satisfies the $(P S)_{c}$ condition.

Corollary 2.9. The functional $\Phi_{\varepsilon}$ given in Proposition 2.5 satisfies the $(P S)_{c}$ condition on $\widetilde{S}_{\varepsilon}$ for any level $c \in \mathbb{R}$.

Proof. Let $\left\{w_{n}\right\} \subset \widetilde{S}_{\varepsilon}$ be a $(P S)_{c}$ sequence for $\Phi_{\varepsilon}$. Then, $\Phi_{\varepsilon}\left(w_{n}\right) \rightarrow c$ and $\left\|\Phi_{\varepsilon}^{\prime}\left(w_{n}\right)\right\|_{\star} \rightarrow 0$, where $\|\cdot\|_{\star}$ denotes the norm in the dual space of $\left(T_{w_{n}} \widetilde{S}_{\varepsilon}\right)^{*}$. By Proposition 2.5-(b), we know $\left\{m_{\varepsilon}\left(w_{n}\right)\right\}$ is a $(P S)_{c}$ sequence for $\mathcal{J}_{\varepsilon}$ in $H_{A_{\varepsilon}}^{1}\left(\mathbb{R}_{+}^{N+1}, \mathbb{C}\right)$. Then, Lemma 2.8 implies that there is a $w \in \widetilde{S}_{\varepsilon}$ such that $m_{\varepsilon}\left(w_{n}\right) \rightarrow m_{\varepsilon}(w)$ in $H_{A_{\varepsilon}}^{1}\left(\mathbb{R}_{+}^{N+1}, \mathbb{C}\right)$ by passing to a subsequence. From Lemma 2.4-(c), we conclude that $w_{n} \rightarrow w$ in $\widetilde{S}_{\varepsilon}$. This completes the proof.

\section{Multiplicity result of the modified problem}

In this section, we prove a multiplicity result for problem (2.10). In what the follows we shall assume that $\delta>0$ small such that $M_{\delta} \subset \mathcal{O}$, where $\mathcal{O}$ is given in (V2). We start by considering the limit problem related to (2.10), that is, the following problem

$$
\begin{cases}-\Delta w+m^{2} w=0 & \text { in } \mathbb{R}_{+}^{N+1} \\ -\frac{\partial w}{\partial y}=V_{0} w+f(|w|) w & \text { in } \mathbb{R}^{N}=\partial \mathbb{R}_{+}^{N+1}\end{cases}
$$

The solutions of equation (3.1) are critical points of the functional given as

$$
\begin{aligned}
J_{0}(w)= & \frac{1}{2} \int_{\mathbb{R}_{+}^{N+1}}\left(\left|\nabla_{x} w\right|^{2}+\left|\frac{\partial w}{\partial y}\right|^{2}+m^{2} w^{2}\right) d x d y \\
& -\frac{1}{2} \int_{\mathbb{R}^{N}} V_{0}|y(w)|^{2} d x-\int_{\mathbb{R}^{N}} F(|y(w)|) d x
\end{aligned}
$$

Next, we define the Nehari manifold $\mathcal{N}_{0}$ related to $\mathcal{J}_{0}$. We say $w \in \mathcal{N}_{0}$ means $w \in H^{1}\left(\mathbb{R}_{+}^{N+1}\right)$ and satisfies

$$
\int_{\mathbb{R}_{+}^{N+1}}\left(\left|\nabla_{x} w\right|^{2}+\left|\frac{\partial w}{\partial y}\right|^{2}+m^{2} w^{2}\right) d x d y-\int_{\mathbb{R}^{N}} V_{0}|y(w)|^{2} d x=\int_{\mathbb{R}^{N}} f(|y(w)|)|y(w)|^{2} d x .
$$

We denote by $\widetilde{H}^{1}\left(\mathbb{R}_{+}^{N+1}\right)$ the open subset of $H^{1}\left(\mathbb{R}_{+}^{N+1}\right)$ given by

$$
\widetilde{H}^{1}\left(\mathbb{R}_{+}^{N+1}\right)=\left\{w \in H^{1}\left(\mathbb{R}_{+}^{N+1}\right):|\operatorname{supp}| y(w)||>0\right\},
$$

and $\widetilde{S}_{0}=S_{0} \cap \widetilde{H}^{1}\left(\mathbb{R}_{+}^{N+1}\right)$, where $S_{0}$ is the unit sphere of $H^{1}\left(\mathbb{R}_{+}^{N+1}\right)$. As in Section 2, $\widetilde{S}_{0}$ is a non-complete $C^{1,1}$. manifold of codimension 1 , modeled on $H^{1}\left(\mathbb{R}_{+}^{N+1}\right)$ and contained in the open $\widetilde{H}^{1}\left(\mathbb{R}_{+}^{N+1}\right)$. Then, $H^{1}\left(\mathbb{R}_{+}^{N+1}\right)=$ $T_{w} \widetilde{S}_{0} \bigoplus \mathbb{R} w$ for each $w \in \widetilde{S}_{0}$, where

$$
T_{w} \widetilde{S}_{0}=\left\{v \in H^{1}\left(\mathbb{R}_{+}^{N+1}\right):\langle w, v\rangle_{H^{1}}=0\right\} .
$$

In the sequel, we state without proof of the following Lemma 3.1 and Proposition 3.2. The proofs are similar to those of Lemma 2.4 and Proposition 2.5. 
Lemma 3.1. Assume that $(V 1)$ and $(f 1)-(f 4)$ are satisfied. Then the following properties hold:

(a) For each $w \in \widetilde{H}^{1}\left(\mathbb{R}_{+}^{N+1}\right)$, let $h_{w}: \mathbb{R}^{+} \rightarrow \mathbb{R}$ be defined as $h_{w}(s)=\mathcal{J}_{0}(s w)$. Then there exists a unique $s_{w}>0$ such that $h_{w}^{\prime}(s)>0$ in $\left(0, s_{w}\right)$ and $h_{w}^{\prime}(s)<0$ in $\left(s_{w}, \infty\right)$.

(b) There is a $t>0$ independent of $w$ such that $s_{w}>t$ for all $w \in \widetilde{S}_{0}$. Moreover, for each compact set $\mathcal{W} \subset \widetilde{S}_{0}$, there is $C_{\mathcal{W}}>0$ such that $s_{w} \leq C_{\mathcal{W}}$ for all $w \in \mathcal{W}$.

(c) The map $\widehat{m}: \widetilde{S}_{0} \rightarrow \mathcal{N}_{0}$ given by $\widehat{m}(w)=s_{w} w$ is continuous and $m:=\left.\widehat{m}\right|_{\widetilde{S}_{\varepsilon}}$ is a homeomorphism between $\widetilde{S}_{0}$ and $\mathcal{N}_{0}$. Moreover, $m^{-1}(w)=\frac{w}{\|w\|_{H^{1}}}$.

(d) If there is a sequence $\left\{w_{n}\right\} \subset \widetilde{S}_{0}$ such that dist $\left(w_{n}, \partial \widetilde{S}_{0}\right) \rightarrow 0$, then $\left\|m\left(w_{n}\right)\right\|_{H^{1}} \rightarrow \infty$ and $\mathcal{J}_{0}\left(m\left(w_{n}\right)\right) \rightarrow \infty$.

Proposition 3.2. Assume that assumptions (V1) and $(f 1)$ - $(f 4)$ are satisfied. Then

(a) $\widehat{\Phi}_{0} \in C^{1}\left(\widetilde{H}^{1}, \mathbb{R}\right)$ and $\Phi_{0} \in C^{1}\left(\widetilde{S}_{0}, \mathbb{R}\right)$.

(b) If $\left\{w_{n}\right\}$ is a $(P S)_{c}$ sequence of $\Phi_{0}$, then $\left\{m\left(w_{n}\right)\right\}$ is a $(P S)_{c}$ sequence of $\mathcal{J}_{0}$. If $\left\{w_{n}\right\} \subset \mathcal{N}_{0}$ is a bounded $(P S)_{c}$ sequence of $\mathcal{J}_{0}$, then $\left\{m^{-1}\left(w_{n}\right)\right\}$ is a $(P S)_{c}$ sequence of $\Phi_{0}$.

(c) $w$ is a critical point of $\Phi_{0}$ if and only if $m(w)$ is a critical point of $\mathcal{J}_{0}$. Moreover, we have

$$
\inf _{\widetilde{S}_{0}} \Phi_{0}=\inf _{\mathcal{N}_{0}} \mathcal{J}_{0}
$$

As in the previous section, we have the following variational characterization of the infimum of $\mathcal{J}_{0}$ under $\mathcal{N}_{0}$ :

$$
c_{0}=\inf _{w \in \mathcal{N}_{0}} \mathcal{J}_{0}=\inf _{w \in \widetilde{H}^{1}} \max _{t>0} \mathcal{J}_{0}(t w)=\inf _{w \in \widetilde{S}_{0}} \max _{t>0} \mathcal{J}_{0}(t w)>0 .
$$

The next lemma allows us to assume the weak limit of a $(P S)_{c}$ sequence is nontrivial.

Lemma 3.3. Let $\left\{w_{n}\right\} \subset H^{1}\left(\mathbb{R}_{+}^{N+1}\right)$ be a $(P S)_{c}$ sequence for $\mathcal{J}_{0}$ for any level $c \in \mathbb{R}$ and such that $w_{n} \rightarrow 0$ weakly in $H^{1}\left(\mathbb{R}_{+}^{N+1}\right)$. Then, one of the following alternatives holds.

(a) $w_{n} \rightarrow 0$ strongly in $H^{1}\left(\mathbb{R}_{+}^{N+1}\right)$,or

(b) there exists a sequence $\left\{z_{n}\right\} \subset \mathbb{R}^{N}$ and constants $R>0$ and $\beta>0$ such that

$$
\liminf _{n \rightarrow \infty} \int_{B_{R}\left(z_{n}\right)}\left|y\left(w_{n}\right)\right|^{2} d x \geq \beta>0 .
$$

Proof. Suppose the case (b) doesn't true. It follows that for all $R>0$, we have

$$
\lim _{n \rightarrow \infty} \sup _{z \in \mathbb{R}^{N}} \int_{B_{R}(z)}\left|y\left(w_{n}\right)\right|^{2} d x=0 .
$$

Due to Lions' Lemma (see for example [36]), we have

$$
y\left(w_{n}\right) \rightarrow 0 \quad \text { in } L^{q}\left(\mathbb{R}^{N}\right) \text { for } 2<q<2^{\sharp} .
$$

Therefore, by (f1)-(f2), we have

$$
\int_{\mathbb{R}^{N}} f\left(\left|y\left(w_{n}\right)\right|\right)\left|y\left(w_{n}\right)\right|^{2} d x \rightarrow 0 .
$$

Since $\left\langle\mathcal{J}_{0}^{\prime}\left(w_{n}\right), w_{n}\right\rangle \rightarrow 0$ as $n \rightarrow \infty$, that is,

$$
\int_{\mathbb{R}_{+}^{N+1}}\left(\left|\nabla_{x} w_{n}\right|^{2}+\left|\frac{\partial w_{n}}{\partial y}\right|^{2}+m^{2} w_{n}^{2}\right) d x d y=\int_{\mathbb{R}^{N}} V_{0}\left|y\left(w_{n}\right)\right|^{2} d x+o_{n}(1) .
$$

Combining this equality and (2.8), we have

$$
\left(1-\frac{V_{0}}{m}\right) \int_{\mathbb{R}_{+}^{N+1}}\left(\left|\nabla_{x} w_{n}\right|^{2}+\left|\frac{\partial w_{n}}{\partial y}\right|^{2}\right) d x d y+m\left(m-V_{0}\right) \int_{\mathbb{R}_{+}^{N+1}} w_{n}^{2} d x d y=o_{n}(1),
$$

which implies that $\left\|w_{n}\right\|_{H^{1}} \rightarrow 0$ strongly and this lemma proved. 
Next, we can obtain a nontrivial ground state solution of equation (3.1).

Theorem 3.4. Let $\left\{w_{n}\right\} \subset H^{1}\left(\mathbb{R}_{+}^{N+1}\right)$ be a $(P S)_{c}$ sequence for $\mathcal{J}_{0}$ for any level $c \in \mathbb{R}$. Then problem (3.1) has a nontrivial ground state solution.

Proof. By Lemma 2.6, we know that $\left\{w_{n}\right\}$ is bounded in $H^{1}\left(\mathbb{R}_{+}^{N+1}\right)$. Then, up to a subsequence, $w_{n} \rightarrow w$ weakly in $H^{1}\left(\mathbb{R}_{+}^{N+1}\right)$ and $y\left(w_{n}\right) \rightarrow y(w)$ in $L_{l o c}^{p}\left(\mathbb{R}^{N}\right)$ and $y\left(w_{n}\right) \rightarrow y(w)$ a.e. in $\mathbb{R}^{N}$. By Lemma 3.3, we know that problem (3.1) has a nontrivial ground state.

The next result is a compactness result of problem (3.1) which will be used later.

Lemma 3.5. Let $\left\{w_{n}\right\} \subset \mathcal{N}_{0}$ be such that $\mathcal{J}_{0}\left(w_{n}\right) \rightarrow c_{0}$. Then $\left\{w_{n}\right\}$ has a convergent subsequence in $H^{1}\left(\mathbb{R}_{+}^{N+1}\right)$.

Proof. Since $\left\{w_{n}\right\} \subset \mathcal{N}_{0}$, it follows from Lemma 3.1-(c), Proposition 3.2-(c) and the definition of $c_{0}$ in (3.3) that

$$
v_{n}=m^{-1}\left(w_{n}\right)=\frac{w_{n}}{\left\|w_{n}\right\|_{H^{1}}} \in \widetilde{S}_{0}, \quad n \in \mathbb{N}
$$

and

$$
\Phi_{0}\left(v_{n}\right)=\mathcal{J}_{0}\left(w_{n}\right) \rightarrow c_{0}=\inf _{w \in \widetilde{S}_{0}} \Phi_{0}(w) .
$$

Although $\widetilde{S}_{0}$ is not complete, by Lemma 3.1-(d) we can still use the Ekeland's variational principle [37] to the functional $\varepsilon_{0}: H \rightarrow \mathbb{R} \cup\{\infty\}$, given as $\mathcal{E}_{0}(w):=\widehat{\Phi}_{0}(w)$ if $w \in \widetilde{S}_{0}$ and $\varepsilon_{0}(w):=\infty$ if $w \in \partial \widetilde{S}_{0}$, where $H=\widetilde{\widetilde{S}}_{0}$ is the complete metric space equipped with the metric $d(w, v):=\|w-v\|_{H^{1}}$. In fact, by Lemma 3.1-(d), $\varepsilon_{0} \in C(H, \mathbb{R} \cup\{\infty\})$ and $\varepsilon_{0}$ is bounded below by Proposition 3.2-(c). Therefore, we can conclude that there is a sequence $\left\{\widetilde{v}_{n}\right\} \subset \widetilde{S}_{0}$ such that $\left\{\widetilde{v}_{n}\right\}$ is a $(P S)_{c_{0}}$ sequence for $\Phi_{0}$ on $\widetilde{S}_{0}$ and

$$
\left\|w_{n}-\widetilde{v}_{n}\right\|_{H^{1}} \rightarrow 0 \text { as } n \rightarrow \infty \text {. }
$$

We conclude the result by applying Proposition 3.2, Theorem 3.4 and processing a similar argument as the proof of Corollary 2.9.

In the following, we will relate the number of nontrivial solution of (2.10). So we consider $\delta>0$ such that $M_{\delta} \subset \mathcal{O}$ and choose $\eta \in C_{0}^{\infty}\left(\mathbb{R}^{+},[0,1]\right)$ satisfying $\eta \equiv 1$ in $\left[0, \frac{\delta}{2}\right]$ and $\eta \equiv 0$ in $[\delta, \infty)$.

For any $z \in M$, we define

$$
\Psi_{\varepsilon, z}(x, y):=\eta(|(\varepsilon x-z, y)|) w\left(\frac{\varepsilon x-z}{\varepsilon}, y\right) \exp \left(i \tau_{z}\left(\frac{\varepsilon x-z}{\varepsilon}, y\right)\right),
$$

where

$$
\tau_{z}(x, y)=\tilde{A}(x, y) \cdot(x, y)=(A(x), 0) \cdot(x, y)=\sum_{j=1}^{N} A_{j}(x) x_{j},
$$

and $s_{\varepsilon}>0$ such that

$$
\max _{s \geq 0} \mathcal{J}_{\varepsilon}\left(s \Psi_{\varepsilon, z}\right)=\mathcal{J}_{\varepsilon}\left(s_{\varepsilon} \Psi_{\varepsilon, z}\right)
$$

where $w$ is a solution of (3.1) from Theorem 3.4 satisfying $\mathcal{J}_{0}(w)=c_{0}$.

Let $\Theta_{\varepsilon}: M \rightarrow \mathcal{N}_{\varepsilon}$ be as

$$
\Theta_{\varepsilon}(z)=s_{\varepsilon} \Psi_{\varepsilon, z} .
$$

The energy of the above function has the following behavior:

Lemma 3.6. Uniformly for $z \in M$, we have

$$
\lim _{\varepsilon \rightarrow 0} \mathcal{J}_{\mathcal{E}}\left(\Theta_{\varepsilon}(z)\right)=c_{0}
$$


Proof. We prove the lemma by contradiction arguments and assume that there is some $\delta_{0}>0,\left\{z_{n}\right\} \subset M$ and $\varepsilon_{n} \rightarrow 0$ such that

$$
\left|\mathcal{J}_{\mathcal{E}_{n}}\left(\Theta_{\varepsilon_{n}}\left(z_{n}\right)\right)-c_{0}\right| \geq \delta_{0} .
$$

Observe that for each $n \in \mathbb{N}$ and for all $x^{\prime} \in B_{\delta / \varepsilon_{n}}(0)$, we have $\varepsilon_{n} x^{\prime} \in B_{\delta}(0)$. Then, we have

$$
\varepsilon_{n} x^{\prime}+z_{n} \in B_{\delta}\left(z_{n}\right) \subset M_{\delta} \subset \mathcal{O} .
$$

By using the change of variable $x^{\prime}:=\left(\varepsilon_{n} x-z_{n}\right) / \varepsilon_{n}$, we can write

$$
\begin{aligned}
\mathcal{J}_{\varepsilon_{n}}\left(\Theta_{\varepsilon_{n}}\left(z_{n}\right)\right)= & \frac{s_{\varepsilon_{n}}^{2}}{2}\left(\int_{\mathbb{R}_{+}^{N+1}}\left(\left|\nabla_{x} \Psi_{\varepsilon_{n}, z_{n}}\right|^{2}+\left|\frac{\partial \Psi_{\varepsilon_{n}, z_{n}}}{\partial y}\right|^{2}+m^{2} \Psi_{\varepsilon_{n}, z_{n}}^{2}\right) d x d y\right. \\
& \left.+\int_{\mathbb{R}^{N}} V_{\varepsilon_{n}}\left|y\left(\Psi_{\varepsilon_{n}, z_{n}}\right)\right|^{2} d x\right)-\int_{\mathbb{R}^{N}} G_{\varepsilon_{n}}\left(x, s_{\varepsilon_{n}}\left|y\left(\Psi_{\varepsilon_{n}, z_{n}}\right)\right|\right) d x \\
= & \frac{s_{\varepsilon_{n}}^{2}}{2}\left(\int_{\mathbb{R}_{+}^{N+1}}\left(\left|\nabla_{x} \Psi_{\varepsilon_{n}, z_{n}}\right|^{2}+\left|\frac{\partial \Psi_{\varepsilon_{n}, z_{n}}}{\partial y}\right|^{2}+m^{2} \Psi_{\varepsilon_{n}, z_{n}}^{2}\right) d x d y\right. \\
& \left.+\int_{\mathbb{R}^{N}} V_{\varepsilon_{n}}\left|y\left(\Psi_{\varepsilon_{n}, z_{n}}\right)\right|^{2} d x\right)-\int_{\mathbb{R}^{N}} F\left(s_{\varepsilon_{n}}\left|y\left(\eta\left(\left|\left(\varepsilon_{n} x^{\prime}, 0\right)\right|\right) w\left(x^{\prime}, 0\right)\right)\right|\right) d x^{\prime} .
\end{aligned}
$$

Since $\Theta_{\varepsilon_{n}}\left(z_{n}\right) \in \mathcal{N}_{\varepsilon}$ and $g=f$ in $\mathcal{O}$, we have

$$
\begin{aligned}
& s_{\varepsilon_{n}}^{2}\left(\int_{\mathbb{R}_{+}^{N+1}}\left(\left|\nabla_{x} \Psi_{\varepsilon_{n}, z_{n}}\right|^{2}+\left|\frac{\partial \Psi_{\varepsilon_{n}, z_{n}}}{\partial y}\right|^{2}+m^{2} \Psi_{\varepsilon_{n}, z_{n}}^{2}\right) d x d y+\int_{\mathbb{R}^{N}} V_{\varepsilon_{n}}\left|y\left(\Psi_{\varepsilon_{n}, z_{n}}\right)\right|^{2} d x\right) \\
& =\int_{\mathbb{R}^{N}} g_{\varepsilon_{n}}\left(x, s_{\varepsilon_{n}}\left|y\left(\Psi_{\varepsilon_{n}, z_{n}}\right)\right|\right)\left|s_{\varepsilon_{n}} y\left(\Psi_{\varepsilon_{n}, z_{n}}\right)\right|^{2} d x \\
& =\int_{\mathbb{R}^{N}} f\left(s_{\varepsilon_{n}}\left|y\left(\eta\left(\left|\left(\varepsilon_{n} x^{\prime}, 0\right)\right|\right) w\left(x^{\prime}, 0\right)\right)\right|\right)\left|s_{\varepsilon_{n}} y\left(\eta\left(\left|\left(\varepsilon_{n} x^{\prime}, 0\right)\right|\right) w\left(x^{\prime}, 0\right)\right)\right|^{2} d x^{\prime},
\end{aligned}
$$

that is,

$$
\begin{aligned}
& \int_{\mathbb{R}_{+}^{N+1}}\left(\left|\nabla_{x} \Psi_{\varepsilon_{n}, z_{n}}\right|^{2}+\left|\frac{\partial \Psi_{\varepsilon_{n}, z_{n}}}{\partial y}\right|^{2}+m^{2} \Psi_{\varepsilon_{n}, z_{n}}^{2}\right) d x d y+\int_{\mathbb{R}^{N}} V_{\varepsilon_{n}}\left|y\left(\Psi_{\varepsilon_{n}, z_{n}}\right)\right|^{2} d x \\
& =\int_{\mathbb{R}^{N}} f\left(s_{\varepsilon_{n}}\left|y\left(\eta\left(\left|\left(\varepsilon_{n} x^{\prime}, 0\right)\right|\right) w\left(x^{\prime}, 0\right)\right)\right|\right)\left|y\left(\eta\left(\left|\left(\varepsilon_{n} x^{\prime}, 0\right)\right|\right) w\left(x^{\prime}, 0\right)\right)\right|^{2} d x^{\prime} .
\end{aligned}
$$

If $s_{\varepsilon_{n}} \rightarrow \infty$, by the boundedness of the $\Psi_{\varepsilon_{n}, z_{n}}$ and (f4), we have

$$
\begin{aligned}
& \int_{\mathbb{R}_{+}^{N+1}}\left(\left|\nabla_{x} \Psi_{\varepsilon_{n}, z_{n}}\right|^{2}+\left|\frac{\partial \Psi_{\varepsilon_{n}, z_{n}}}{\partial y}\right|^{2}+m^{2} \Psi_{\varepsilon_{n}, z_{n}}^{2}\right) d x d y+\int_{\mathbb{R}^{N}} V_{\varepsilon_{n}}\left|y\left(\Psi_{\varepsilon_{n}, z_{n}}\right)\right|^{2} d x \\
& =\int_{\mathbb{R}^{N}} f\left(s_{\varepsilon_{n}}\left|y\left(\eta\left(\left|\left(\varepsilon_{n} x^{\prime}, 0\right)\right|\right) w\left(x^{\prime}, 0\right)\right)\right|\right)\left|y\left(\eta\left(\left|\left(\varepsilon_{n} x^{\prime}, 0\right)\right|\right) w\left(x^{\prime}, 0\right)\right)\right|^{2} d x^{\prime} \\
& \geq \int_{B_{\delta / 2}(0)} f\left(s_{\varepsilon_{n}}\left|y\left(w\left(x^{\prime}, 0\right)\right)\right|\right)\left|y\left(w\left(x^{\prime}, 0\right)\right)\right|^{2} d x^{\prime} \\
& \geq f\left(s_{\varepsilon_{n}} a\right) \int_{B_{\delta / 2}(0)}\left|y\left(w\left(x^{\prime}, 0\right)\right)\right|^{2} d x^{\prime} \rightarrow \infty
\end{aligned}
$$


as $n \rightarrow \infty$, where $a=\inf _{x \in B_{\delta / 2}(0)}\left|y\left(w\left(x^{\prime}, 0\right)\right)\right|$. This yields a contradiction. Therefore, $s_{\varepsilon_{n}} \rightarrow s_{0} \geq 0$.

By (3.7), (2.8) and (f1), we can get $s_{0}>0$. Next, we claim that $s_{0}=1$. Indeed, by applying the Dominated Convergence Theorem and taking a similar argument as Lemma 3.2 in [17], we have

$$
\begin{gathered}
\int_{\mathbb{R}_{+}^{N+1}}\left(\left|\nabla_{x} \Psi_{\varepsilon_{n}, z_{n}}\right|^{2}+\left|\frac{\partial \Psi_{\varepsilon_{n}, z_{n}}}{\partial y}\right|^{2}+m^{2} \Psi_{\varepsilon_{n}, z_{n}}^{2}\right) d x d y \\
\rightarrow \int_{\mathbb{R}_{+}^{N+1}}\left(\left|\nabla_{x} w\right|^{2}+\left|\frac{\partial w}{\partial y}\right|^{2}+m^{2} w^{2}\right) d x d y, \\
\int_{\mathbb{R}^{N}} V_{\varepsilon_{n}}\left|y\left(\Psi_{\varepsilon_{n}, z_{n}}\right)\right|^{2} d x \rightarrow-V_{0} \int_{\mathbb{R}^{N}}|y(w)|^{2} d x, \\
\int_{\mathbb{R}^{N}} f\left(\left|y\left(\Psi_{\varepsilon_{n}, z_{n}}\right)\right|\right)\left|\Psi_{\varepsilon_{n}, z_{n}}\right|^{2} d x \rightarrow \int_{\mathbb{R}^{N}} f(|y(w)|)|y(w)|^{2} d x .
\end{gathered}
$$

Therefore, by passing the limit in (3.7), we can obtain that

$$
\int_{\mathbb{R}_{+}^{N+1}}\left(|\nabla x w|^{2}+\left|\frac{\partial w}{\partial y}\right|^{2}+m^{2} w^{2}\right) d x d y-V_{0} \int_{\mathbb{R}^{N}}|y(w)|^{2} d x=\int_{\mathbb{R}^{N}} f\left(s_{0}|y(w)|\right)|y(w)|^{2} d x .
$$

On the other hand, since $w$ is a solution of (3.1), we have

$$
\int_{\mathbb{R}_{+}^{N+1}}\left(\left|\nabla_{x} w\right|^{2}+\left|\frac{\partial w}{\partial y}\right|^{2}+m^{2} w^{2}\right) d x d y-V_{0} \int_{\mathbb{R}^{N}}|y(w)|^{2} d x=\int_{\mathbb{R}^{N}} f(|y(w)|)|y(w)|^{2} d x .
$$

Combining (3.11)-(3.12), we have

$$
\int_{\mathbb{R}^{N}}\left[f\left(s_{0}|y(w)|\right)-f(|y(w)|)\right]|y(w)|^{2} d x=0 .
$$

By (f4), we know $s_{0}=1$ and the claim is proved.

Finally, let $n \rightarrow \infty$ in (3.6), we have

$$
\lim _{n \rightarrow \infty} \mathcal{J}_{\mathcal{E}_{n}}\left(\Theta_{\varepsilon_{n}}\left(z_{n}\right)\right)=\mathcal{J}_{0}(w)=c_{0},
$$

which contradicts to (3.5). This completes the proof.

For the $\delta>0$ given before Lemma 3.6, choose $\rho=\rho(\delta)>0$ such that $M_{\delta} \subset B_{\rho}(0)$. Define $\chi: \mathbb{R}^{N} \rightarrow \mathbb{R}^{N}$ as $\chi(x)=x$ if $|x| \leq \rho$ and $\chi(x)=\rho \frac{x}{|x|}$ if $|x| \geq \rho$. Then let us consider the barycenter map $\beta_{\varepsilon}: \mathcal{N}_{\varepsilon} \rightarrow \mathbb{R}^{N}$ given by

$$
\beta_{\varepsilon}(w)=\frac{\int_{\mathbb{R}^{N}} \chi(\varepsilon x)|y(w)|^{2} d x}{\int_{\mathbb{R}^{N}}|y(w)|^{2} d x} .
$$

Since $\mathcal{O} \subset B_{\rho}(0)$, by the definition of $\chi$ and Lebesgue's Theorem, we conclude that

$$
\lim _{\varepsilon \rightarrow 0} \beta_{\varepsilon}\left(\Theta_{\varepsilon}(z)\right)=z \quad \text { uniformly in } z \in \mathcal{O} .
$$

The next compactness result is fundamental for proving that the solutions of the modified problem are solution of the original problem.

Proposition 3.7. Let $\varepsilon_{n} \rightarrow 0$ and $\left\{w_{n}\right\} \subset \mathcal{N}_{\varepsilon_{n}}$ be such that $\mathcal{J}_{\mathcal{E}_{n}}\left(w_{n}\right) \rightarrow c_{0}$. Then there exists a sequence $\left\{\tilde{z}_{n}\right\} \subset$ $\mathbb{R}^{N}$ such that $v_{n}(x)=w_{n}\left(x+\tilde{z}_{n}\right)$ has a convergent subsequence in $H^{1}\left(\mathbb{R}_{+}^{N+1}\right)$. Moreover, up to a subsequence, $z_{n}:=\varepsilon_{n} \tilde{z}_{n} \rightarrow z_{0} \in \mathcal{O}$. 
Proof. By Lemma 2.6, we know that $\left\|w_{n}\right\|_{H_{A_{\varepsilon_{n}}}^{1}} \leq C$ for $n \in \mathbb{N}$. Note that $c_{0}>0$, and since $\left\|w_{n}\right\|_{{H_{A_{n}}}_{1}^{1}} \rightarrow 0$ would imply $\mathcal{J}_{\mathcal{E}_{n}}\left(w_{n}\right) \rightarrow 0$, we can argue as in Lemma 3.3 to get a subsequence $\left\{\tilde{z}_{n}\right\} \subset \mathbb{R}^{N}$ and constants $R, \beta>0$ such that

$$
\liminf _{n \rightarrow \infty} \int_{B_{R}\left(\tilde{z}_{n}\right)}\left|y\left(w_{n}\right)\right|^{2} d x \geq \beta>0 .
$$

Let $v_{n}(x)=w_{n}\left(x+\tilde{z}_{n}\right)$, then $\left\{v_{n}\right\}$ is also bounded and therefore, along a subsequence, we have $v_{n} \rightarrow v \not \equiv$ weakly in $H^{1}\left(\mathbb{R}_{+}^{N+1}\right)$. Take $t_{n}>0$ such that $\tilde{v}_{n}:=t_{n} v_{n} \in \mathcal{N}_{0}$, and set $z_{n}=\varepsilon_{n} \tilde{z}_{n}$. Since $w_{n} \in \mathcal{N}_{\varepsilon_{n}}$, we have

$$
\begin{aligned}
c_{0} \leq \mathcal{J}_{0}\left(\tilde{v}_{n}\right) \leq & \frac{1}{2} \int_{\mathbb{R}_{+}^{N+1}}\left(\left|\nabla_{x} \tilde{v}_{n}\right|^{2}+\left|\frac{\partial \tilde{v}_{n}}{\partial y}\right|^{2}+m^{2} \tilde{v}_{n}^{2}\right) d x d y \\
& +\frac{1}{2} \int_{\mathbb{R}^{N}} V\left(\varepsilon_{n} x+z_{n}\right)\left|y\left(\tilde{v}_{n}\right)\right|^{2} d x-\int_{\mathbb{R}^{N}} F\left(\left|y\left(\tilde{v}_{n}\right)\right|\right) d x \\
\leq & \frac{t_{n}^{2}}{2} \int_{\mathbb{R}_{+}^{N+1}}\left(\left|\nabla_{x} w_{n}\right|^{2}+\left|\frac{\partial w_{n}}{\partial y}\right|^{2}+m^{2} w_{n}^{2}\right) d x d y \\
& +\frac{t_{n}^{2}}{2} \int_{\mathbb{R}^{N}} V\left(\varepsilon_{n} x\right)\left|y\left(w_{n}\right)\right|^{2} d x-\int_{\mathbb{R}^{N}} G\left(\varepsilon_{n} x, t_{n}\left|y\left(w_{n}\right)\right|\right) d x \\
= & \mathcal{J}_{\varepsilon_{n}}\left(t_{n} w_{n}\right) \\
\leq & \mathcal{J}_{\varepsilon_{n}}\left(w_{n}\right)=c_{0}+o_{n}(1),
\end{aligned}
$$

where implies $\lim _{n \rightarrow \infty} \mathcal{J}_{0}\left(\tilde{v}_{n}\right)=c_{0}$. Moreover, $\left\{\tilde{v}_{n}\right\}$ is bounded in $H^{1}\left(\mathbb{R}_{+}^{N+1}\right)$ and $\tilde{v}_{n} \rightarrow \tilde{v}$. We may assume that $t_{n} \rightarrow t^{\star}>0$. By the uniqueness of the weak limit, we have that $\tilde{v}=t^{\star} v \not \equiv 0$. By Lemma 3.5, $\tilde{v}_{n} \rightarrow \tilde{v}$ in $H^{1}\left(\mathbb{R}_{+}^{N+1}\right)$, and thus $v_{n} \rightarrow v$ in $H^{1}\left(\mathbb{R}_{+}^{N+1}\right)$. Moreover,

$$
\mathcal{J}_{0}(\tilde{v})=c_{0} \quad \text { and }\left\langle\mathcal{J}_{0}^{\prime}(\tilde{v}), \tilde{v}\right\rangle=0 .
$$

Next, we prove that $\left\{z_{n}\right\}$ has a bounded subsequence. In fact, suppose by contradiction that $\left|z_{n}\right| \rightarrow \infty$. Choose $R>0$ such that $\mathcal{O} \subset B_{R}(0)$. Then for $n$ large enough, we have $\left|z_{n}\right|>2 R$ and for each $x \in B_{R / \varepsilon_{n}}(0)$ we have

$$
\left|\varepsilon_{n} x+z_{n}\right| \geq\left|z_{n}\right|-\left|\varepsilon_{n} x\right| \geq 2 R-R=R .
$$

Therefore, by $v_{n} \rightarrow v$ in $H^{1}\left(\mathbb{R}_{+}^{N+1}\right)$, the above expression, the definition of $g$ and Lebesgue's theorem, we can get

$$
\begin{aligned}
& \int_{\mathbb{R}_{+}^{N+1}}\left(\left|\nabla_{x} v_{n}\right|^{2}+\left|\frac{\partial v_{n}}{\partial y}\right|^{2}+m^{2} v_{n}^{2}\right) d x d y-\int_{\mathbb{R}^{N}} V_{0}\left|y\left(v_{n}\right)\right|^{2} d x \\
& \leq \int_{\mathbb{R}^{N}} g\left(\varepsilon_{n} x+z_{n},\left|y\left(v_{n}\right)\right|\right)\left|y\left(v_{n}\right)\right|^{2} d x \\
& \quad=\int_{B_{R / \varepsilon_{n}}(0)} \tilde{f}\left(\left|y\left(v_{n}\right)\right|\right)\left|y\left(v_{n}\right)\right|^{2} d x+\int_{\mathbb{R}^{N} \backslash B_{R / \varepsilon_{n}}(0)} g\left(\varepsilon_{n} x+z_{n},\left|y\left(v_{n}\right)\right|\right)\left|y\left(v_{n}\right)\right|^{2} d x \\
& \leq \int_{B_{R / \varepsilon_{n}}(0)} \tilde{f}\left(\left|y\left(v_{n}\right)\right|\right)\left|y\left(v_{n}\right)\right|^{2} d x+\int_{\mathbb{R}^{N} \backslash B_{R / \varepsilon_{n}}(0)} f\left(\left|y\left(v_{n}\right)\right|\right)\left|y\left(v_{n}\right)\right|^{2} d x \\
& \leq \frac{V_{0}}{K} \int_{\mathbb{R}^{N}}\left|y\left(v_{n}\right)\right|^{2} d x+o_{n}(1) .
\end{aligned}
$$

Together (3.16) and (2.8), we have

$$
\left(1-\left(1+\frac{1}{K}\right) \frac{V_{0}}{m}\right) \int_{\mathbb{R}_{+}^{N+1}}\left(\left|\nabla_{x} V_{n}\right|^{2}+\left|\frac{\partial v_{n}}{\partial y}\right|^{2}\right) d x d y
$$




$$
\begin{aligned}
& \quad+m\left(m-\left(1+\frac{1}{K}\right) V_{0}\right) \int_{\mathbb{R}_{+}^{N+1}}\left|v_{n}\right|^{2} d x d y \\
& \leq o_{n}(1) .
\end{aligned}
$$

Then, we have $v_{n} \rightarrow 0$ in $H^{1}\left(\mathbb{R}_{+}^{N+1}\right)$, which contradicts with $v \not \equiv 0$. So $\left\{z_{n}\right\}$ is bounded and we can assume that $z_{n} \rightarrow z_{0} \in \mathbb{R}^{N}$. If $z_{0} \notin \overline{\mathcal{O}}$, we can proceed as above to conclude that $v_{n} \rightarrow 0$. Then, we have that $z_{0} \in \overline{\mathcal{O}}$.

Finally, we show that $V\left(z_{0}\right)=-V_{0}$, we can suppose by contradiction that $-V_{0}<V\left(z_{0}\right)$. Then, by $\tilde{v}_{n} \rightarrow \tilde{v}$ in $H^{1}\left(\mathbb{R}_{+}^{N+1}\right)$, Fatou's Lemma and the invariance of $\mathbb{R}^{N}$ by translation, we have

$$
\begin{aligned}
c_{0}=\mathcal{J}_{0}(\tilde{v})< & \frac{1}{2} \int_{\mathbb{R}_{+}^{N+1}}\left(\left|\nabla_{x} \tilde{v}\right|^{2}+\left|\frac{\partial \tilde{v}}{\partial y}\right|^{2}+m^{2} \tilde{v}^{2}\right) d x d y \\
& +\frac{1}{2} \int_{\mathbb{R}^{N}} V\left(z_{0}\right)|y(\tilde{v})|^{2} d x-\int_{\mathbb{R}^{N}} F(|y(\tilde{v})|) d x \\
= & \frac{1}{2} \int_{\mathbb{R}_{+}^{N+1}}\left(\left|\nabla_{x} \tilde{v}\right|^{2}+\left|\frac{\partial \tilde{v}}{\partial y}\right|^{2}+m^{2} \tilde{v}^{2}\right) d x d y \\
& +\frac{1}{2} \int_{\mathbb{R}^{N}}\left(V\left(z_{0}\right)+V_{0}\right)|y(\tilde{v})|^{2} d x-\frac{1}{2} \int_{\mathbb{R}^{N}} V_{0}|y(\tilde{v})|^{2} d x-\int_{\mathbb{R}^{N}} F(|y(\tilde{v})|) d x \\
\leq & \liminf _{n \rightarrow \infty}\left\{\frac{1}{2} \int_{\mathbb{R}_{+}^{N+1}}\left(\left|\nabla_{x} \tilde{v}_{n}\right|^{2}+\left|\frac{\partial \tilde{v}_{n}}{\partial y}\right|^{2}+m^{2} \tilde{v}_{n}^{2}\right) d x d y\right. \\
& \left.+\frac{1}{2} \int_{\mathbb{R}^{N}} V\left(\varepsilon_{n} x+z_{n}\right)\left|y\left(\tilde{v}_{n}\right)\right|^{2} d x-\int_{\mathbb{R}^{N}} F\left(\left|y\left(\tilde{v}_{n}\right)\right|\right) d x\right\} \\
= & \liminf _{n \rightarrow \infty}\left\{\frac{t_{n}^{2}}{2} \int_{\mathbb{R}_{+}^{N+1}}\left(\left|\nabla_{x} w_{n}\right|^{2}+\left|\frac{\partial w_{n}}{\partial y}\right|^{2}+m^{2} w_{n}^{2}\right) d x d y\right. \\
& \left.+\frac{t_{n}^{2}}{2} \int_{\mathbb{R}^{N}} V\left(\varepsilon_{n} x\right)\left|y\left(w_{n}\right)\right|^{2} d x-\iint_{\mathbb{R}^{N}} G\left(\varepsilon_{n} x, t_{n}\left|y\left(\tilde{v}_{n}\right)\right|\right) d x\right\} \\
\leq & \liminf _{n \rightarrow \infty} \lim _{n \rightarrow \infty}\left(t_{n} w_{n}\right) \\
\leq & \operatorname{Jim}_{\mathcal{E}_{n}}\left(w_{n}\right)=c_{0},
\end{aligned}
$$

which yields a contradiction. Therefore, $V\left(z_{0}\right)=-V_{0}$ and $z \in \bar{M}$. The condition (V2) implies that $z_{0} \notin \partial M$ and thus $z_{0} \in M$. This completes the proof.

Now, we consider the following subset of the Nehari manifold

$$
\widetilde{\mathcal{N}}_{\varepsilon}=\left\{w \in \mathcal{N}_{\varepsilon}: \mathcal{J}_{\varepsilon}(w) \leq c_{0}+h(\varepsilon)\right\},
$$

where $h: \mathbb{R}^{+} \rightarrow \mathbb{R}^{+}$is such that $h(\varepsilon) \rightarrow 0^{+}$if $\varepsilon \rightarrow 0^{+}$. Given $z \in M$, we can use Lemma 3.6 to get that $h(\varepsilon)=\left|\mathcal{J}_{\varepsilon}\left(\Theta_{\varepsilon}(z)\right)-c_{0}\right|$ is such that $h(\varepsilon) \rightarrow 0$ as $\varepsilon \rightarrow 0^{+}$. Therefore, $\Theta_{\varepsilon}(z) \in \widetilde{\mathcal{N}}_{\varepsilon}$ and $\widetilde{\mathcal{N}}_{\varepsilon} \neq \emptyset$ for any $\varepsilon>0$.

We present below an interesting relation between $\widetilde{\mathcal{N}}_{\varepsilon}$ and the barycenter map.

Lemma 3.8. For each $\delta>0$, there holds that

$$
\lim _{\varepsilon \rightarrow 0} \sup _{w \in \widetilde{\mathcal{N}}_{\varepsilon}} \operatorname{dist}\left(\beta_{\varepsilon}(w), M_{\delta}\right)=0
$$

Proof. Let $\left\{\varepsilon_{n}\right\} \subset \mathbb{R}^{+}$be such that $\varepsilon_{n} \rightarrow 0$, then there exists $\left\{w_{n}\right\} \subset \widetilde{\mathcal{N}}_{\varepsilon}$ such that

$$
\operatorname{dist}\left(\beta_{\varepsilon}\left(w_{n}\right), M_{\delta}\right)=\lim _{\varepsilon \rightarrow 0} \sup _{w \in \widetilde{\mathcal{N}}_{\varepsilon}} \operatorname{dist}\left(\beta_{\varepsilon}(w), M_{\delta}\right)+o_{n}(1) .
$$


So it suffices to find a sequence $\left\{z_{n}\right\} \subset M_{\delta}$ such that

$$
\left|\beta_{\varepsilon}\left(w_{n}\right)-z_{n}\right|=o_{n}(1) .
$$

Since $\mathcal{J}_{0}\left(t w_{n}\right) \leq \mathcal{J}_{\mathcal{E}}\left(t w_{n}\right)$ for all $t \geq 0$ and $\left\{w_{n}\right\} \subset \widetilde{\mathcal{N}}_{\varepsilon_{n}} \subset \mathcal{N}_{\varepsilon_{n}}$, we have

$$
c_{0} \leq c_{\varepsilon_{n}} \leq \mathcal{J}_{\varepsilon_{n}}\left(w_{n}\right) \leq c_{0}+h\left(\varepsilon_{n}\right) .
$$

Then, we can obtain that $\mathcal{J}_{\varepsilon_{n}}\left(w_{n}\right) \rightarrow c_{0}$. So we can invoke Proposition 3.7 to obtain a sequence $\left\{\tilde{z}_{n}\right\} \subset \mathbb{R}^{N}$ such that $z_{n}=\varepsilon_{n} \tilde{z}_{n} \in M_{\delta}$ and $z_{n} \rightarrow z_{0} \in M$. Therefore,

$$
\begin{aligned}
\beta_{\varepsilon_{n}}\left(w_{n}\right) & =\frac{\int_{\mathbb{R}^{N}} \chi\left(\varepsilon_{n} x\right)\left|y\left(w_{n}\right)\right|^{2} d x}{\int_{\mathbb{R}^{N}}\left|y\left(w_{n}\right)\right|^{2} d x} \\
& =\frac{\int_{\mathbb{R}^{N}} \chi\left(\varepsilon_{n} x+z_{n}\right)\left|y\left(w_{n}\right)\right|^{2} d x}{\int_{\mathbb{R}^{N}}\left|y\left(w_{n}\right)\right|^{2} d x} \\
& =z_{n}+\frac{\int_{\mathbb{R}^{N}}\left(\chi\left(\varepsilon_{n} x+z_{n}\right)-z_{n}\right)\left|y\left(w_{n}\right)\right|^{2} d x}{\int_{\mathbb{R}^{N}}\left|y\left(w_{n}\right)\right|^{2} d x} .
\end{aligned}
$$

Since $\varepsilon_{n} x+z_{n} \rightarrow z_{0} \in M_{\delta}$, we see that $\beta_{\varepsilon_{n}}\left(w_{n}\right)=z_{n}+o_{n}(1)$ and thus the sequence $\left\{z_{n}\right\}$ satisfies (3.18) and the lemma is proved.

We finish this section by presenting a relation between the topology of $M$ and the number of solutions of the modified equation (2.10). Since $\widetilde{S}_{\varepsilon}$ is not a complete metric space, we will invoke the abstract category result in [32].

Theorem 3.9. Assume that $(A),(V 1)-(V 2)$ and $(f 1)-(f 4)$ hold. Then for any $\delta>0$, there exists $\hat{\varepsilon}_{\delta}>0$ such that problem (2.10) has at least cat $M_{M_{\delta}}(M)$ solutions provides $\varepsilon \in\left(0, \hat{\varepsilon}_{\delta}\right)$.

Proof. For each $\varepsilon>0$, we define the function $\pi_{\varepsilon}: M \rightarrow \widetilde{S}_{\varepsilon}$ by

$$
\pi_{\varepsilon}(z)=m_{\varepsilon}^{-1}\left(\Theta_{\varepsilon}(z)\right), \quad \text { for any } z \in M .
$$

By Lemma 3.6 and Proposition 2.5-(c), we have

$$
\lim _{\varepsilon \rightarrow 0} \Phi_{\varepsilon}\left(\pi_{\varepsilon}(z)\right)=\lim _{\varepsilon \rightarrow 0} \mathcal{J}_{\varepsilon}\left(\Theta_{\varepsilon}(z)\right)=c_{0}, \quad \text { uniformly in } z \in M .
$$

Therefore, there is number $\hat{\varepsilon}>0$ such that the set

$$
\widehat{\widetilde{S}}_{\varepsilon}=\left\{w \in \widetilde{S}_{\varepsilon}: \Phi_{\varepsilon}(w) \leq c_{0}+h(\varepsilon)\right\}
$$

is nonempty for all $\varepsilon \in(0, \hat{\varepsilon})$ since $\pi_{\varepsilon}(M) \subset \widehat{\widetilde{S}}_{\varepsilon}$. Here $h$ is given in the definition of $\widetilde{N}_{\varepsilon}$.

From the above considerations, together with Lemma 3.6, Lemma 2.4-(c), (3.14) and Lemma 3.8, we see that there exists a $\hat{\varepsilon}=\hat{\varepsilon}_{\delta}>0$ such that the diagram of continuous mappings below is well-defined for $\varepsilon \in(0, \hat{\varepsilon})$

$$
M \stackrel{\Theta_{\varepsilon}}{\longrightarrow} \Theta_{\varepsilon}(M) \stackrel{m_{\varepsilon}^{-1}}{\longrightarrow} \pi_{\varepsilon}(M) \stackrel{m_{\varepsilon}}{\longrightarrow} \Theta_{\varepsilon}(M) \stackrel{\beta_{\varepsilon}}{\longrightarrow} M_{\delta} .
$$

From (3.14), we can choose a function $\tau(\varepsilon, z)$ with $|\tau(\varepsilon, z)|<\delta / 2$ uniformly in $z \in M$ for all $\varepsilon \in(0, \hat{\varepsilon})$, such that $\beta_{\varepsilon}\left(\Theta_{\varepsilon}(z)\right)=z+\tau(\varepsilon, z)$ for all $z \in M$. Define $H(t, z)=z+(1-t) \tau(\varepsilon, z)$. Then $H:[0,1] \times M \rightarrow M_{\delta}$ is continous. Observe that $H(0, z)=\beta_{\varepsilon}\left(\Theta_{\varepsilon}(z)\right), H(1, z)=z$ for all $z \in M$. So $H(t, z)$ is a homotopy between $\beta_{\varepsilon} \circ \Theta_{\varepsilon}=\left(\beta_{\varepsilon} \circ m_{\varepsilon}\right) \circ \pi_{\varepsilon}$ and the inclusion map id $: M \rightarrow M_{\delta}$. Therefore, we have

$$
\operatorname{cat}_{\pi_{\varepsilon}(M)} \pi_{\varepsilon}(M) \geq \operatorname{cat}_{M_{\delta}}(M) .
$$

It follows from Corollary 2.9 and the category abstract theorem (see [32], Corollary 2.8) that $\Phi_{\varepsilon}$ has at least $\mathrm{cat}_{\pi_{\varepsilon}(M)} \pi_{\varepsilon}(M)$ critical points on $\widehat{\widetilde{S}}_{\varepsilon}$ provides $c=c_{\varepsilon} \leq c_{0}+h(\varepsilon)=d$ and $K=\phi_{\varepsilon}(M)$. Then, by Proposition 2.5-(c) and (3.19), we conclude that $\mathcal{J}_{\varepsilon}$ admits at least $c a t_{M_{\delta}}(M)$ critical points in $\widetilde{\mathcal{N}}_{\varepsilon}$. Finally, we know (2.10) has at least $c^{a t} M_{\delta}(M)$ solutions. 


\section{Proof of the main results}

In this section, we will prove of main results. The idea is to show the solutions obtained in Theorem 3.9 satisfy the estimate $w_{\varepsilon}(x) \leq a$ for any $x \in \mathcal{O}_{\varepsilon}^{c}$ as $\varepsilon$ is small. This fact implies that these solutions are indeed solutions of the original problem (2.7). The following lemma plays an important role in the study of behavior of the maximum points of the solutions, whose proof is related to the Morse iterative method [31] (see also [18, 33, 38]).

Lemma 4.1. Let $\varepsilon_{n} \rightarrow 0^{+}$and $w_{n} \in \widetilde{\mathcal{N}}_{\varepsilon_{n}}$ be s solution of (2.10) with $\varepsilon=\varepsilon_{n}$. Then $\mathcal{J}_{\mathcal{E}_{n}}\left(w_{n}\right) \rightarrow c_{0}$ and $v_{n}(x, y)=$ $w_{n}\left(x+\tilde{z}_{n}, y\right)$ satisfies $y\left(v_{n}\right) \in L^{\infty}\left(\mathbb{R}^{N}\right)$. Moreover,

$$
\lim _{|x| \rightarrow \infty} y\left(v_{n}\right)=0 .
$$

Proof. Since $\mathcal{J}_{\varepsilon_{n}}\left(w_{n}\right) \leq c_{0}+h\left(\varepsilon_{n}\right)$ with $\lim _{n \rightarrow \infty} h\left(\varepsilon_{n}\right)=0$, we can argue as in the proof of equation (3.15) to conclude that $\mathcal{J}_{\varepsilon_{n}}\left(w_{n}\right) \rightarrow c_{0}$.

Set

$$
v_{L, n}(x, y):=\min \left\{\left|v_{n}(x, y)\right|, L\right\}, \quad \varphi_{L, n}:=v_{L, n}^{2(\beta-1)} v_{n} .
$$

with $\beta>1$ to be determined later.

Since

$$
\begin{aligned}
\overline{\nabla_{\tilde{A}_{\varepsilon_{n}}} \varphi_{L, n}} & =v_{L, n}^{2(\beta-1)} \overline{\nabla_{\tilde{A}_{\varepsilon_{n}}} v_{n}}+2(\beta-1) v_{L, n}^{2(\beta-1)-1} \nabla\left|v_{n}\right| \chi_{\left\{\left|v_{n}\right|<L\right\}} \overline{v_{n}} \\
& =v_{L, n}^{2(\beta-1)} \overline{\nabla_{\tilde{A}_{\varepsilon_{n}}} v_{n}}+2(\beta-1) v_{L, n}^{2(\beta-1)-1} \nabla v_{L, n} \overline{v_{n}},
\end{aligned}
$$

then

$$
\nabla_{\tilde{A}_{\varepsilon_{n}}} v_{n} \overline{\nabla_{\tilde{A}_{\varepsilon_{n}}} \varphi_{L, n}}=w_{L, n}^{2(\beta-1)}\left|\nabla_{\tilde{A}_{\varepsilon_{n}}} v_{n}\right|^{2}+2(\beta-1) v_{L, n}^{2(\beta-1)-1} \overline{v_{n}} \nabla v_{L, n} \nabla_{\tilde{A}_{\varepsilon_{n}}} v_{n}
$$

By a direct computation (see for example [20]), we have

$$
\Re\left(\overline{v_{n}} \nabla_{\tilde{A}_{\varepsilon_{n}}} v_{n}\right)=\left|v_{n}\right| \nabla\left|v_{n}\right| \text {. }
$$

Therefore, by the diamagnetic inequality, we obtain

$$
\begin{aligned}
\Re\left(\nabla_{\tilde{A}_{\varepsilon_{n}}} v_{n} \overline{\nabla_{\tilde{A}_{\varepsilon_{n}}} \varphi_{L, n}}\right) & \geq v_{L, n}^{2(\beta-1)}|\nabla| v_{n}||^{2}+2(\beta-1) v_{L, n}^{2(\beta-1)-1}\left|v_{n}\right| \nabla v_{L, n} \nabla\left|v_{n}\right| \\
& =\left.v_{L, n}^{2(\beta-1)}|\nabla| v_{n}\right|^{2}+2(\beta-1) v_{L, n}^{2(\beta-1)}\left|\nabla v_{L, n}\right|^{2} .
\end{aligned}
$$

This inequality, by the definition of $\varphi_{L, n}$ and $\left\langle\mathcal{J}_{\varepsilon_{n}}^{\prime}\left(v_{n}\right), \varphi_{L, n}\right\rangle=0$ imply that

$$
\begin{aligned}
& \int_{\mathbb{R}_{+}^{N+1}} v_{L, n}^{2(\beta-1)}|\nabla| v_{n}||^{2} d x d y+2(\beta-1) \int_{\mathbb{R}_{+}^{N+1}} v_{L, n}^{2(\beta-1)}\left|\nabla v_{L, n}\right|^{2} d x d y \\
& \leq \int_{\mathbb{R}^{N}}\left(g_{\varepsilon_{n}}\left(x,\left|y\left(v_{n}\right)\right|\right)-V_{\varepsilon_{n}}(x)\right)\left|y\left(v_{L, n}\right)\right|^{2(\beta-1)}\left|y\left(v_{n}\right)\right|^{2} d x \\
& \quad-m^{2} \int_{\mathbb{R}_{+}^{N+1}} v_{L, n}^{2(\beta-1)} v_{n}^{2} d x d y \\
& \leq \int_{\mathbb{R}^{N}}\left(g_{\varepsilon_{n}}\left(x,\left|y\left(v_{n}\right)\right|\right)+V_{0}(x)\right)\left|y\left(v_{L, n}\right)\right|^{2(\beta-1)}\left|y\left(v_{n}\right)\right|^{2} d x,
\end{aligned}
$$

here we use the fact $V+V_{0} \geq 0$ for all $x \in \mathbb{R}^{n}$ in the last inequality.

By (2.12), then from (4.1) we have

$$
\left.\int_{\mathbb{R}_{+}^{N+1}} v_{L, n}^{2(\beta-1)}|\nabla| v_{n}\right|^{2} d x d y+2(\beta-1) \int_{\mathbb{R}_{+}^{N+1}} v_{L, n}^{2(\beta-1)}\left|\nabla v_{L, n}\right|^{2} d x d y
$$




$$
\leq\left(\varepsilon+V_{0}\right) \int_{\mathbb{R}^{N}}\left|y\left(v_{L, n}\right)\right|^{2(\beta-1)}\left|y\left(v_{n}\right)\right|^{2} d x+C_{\varepsilon} \int_{\mathbb{R}^{N}}\left|y\left(v_{L, n}\right)\right|^{2(\beta-1)}\left|y\left(v_{n}\right)\right|^{2^{\sharp}} d x,
$$

for some $\varepsilon>0$.

On the other hand, let $\omega_{L, n}=v_{L, n}^{\beta-1}\left|v_{n}\right|$ and then

$$
\nabla \omega_{L, n}=v_{L, n}^{\beta-1} \nabla\left|v_{n}\right|+(\beta-1) v_{L, n}^{\beta-2}\left|v_{n}\right| \nabla v_{L, n} .
$$

We deduce from (4.2) and (4.3) that

$$
\begin{aligned}
& \int_{\mathbb{R}_{+}^{N+1}}\left|\nabla \omega_{L, n}\right|^{2} d x d y \\
& \quad \leq C \beta\left(\left.\int_{\mathbb{R}_{+}^{N+1}} v_{L, n}^{2(\beta-1)}|\nabla| v_{n}\right|^{2} d x d y+2(\beta-1) \int_{\mathbb{R}_{+}^{N+1}} v_{L, n}^{2(\beta-1)}\left|\nabla v_{L, n}\right|^{2} d x d y\right) \\
& \quad \leq C \beta\left(\left(\varepsilon+V_{0}\right) \int_{\mathbb{R}^{N}}\left|y\left(v_{L, n}\right)\right|^{2(\beta-1)}\left|y\left(v_{n}\right)\right|^{2} d x+C_{\varepsilon} \int_{\mathbb{R}^{N}}\left|y\left(v_{L, n}\right)\right|^{2(\beta-1)}\left|y\left(v_{n}\right)\right|^{2^{\sharp}} d x\right),
\end{aligned}
$$

for positive constant $C$. By the Sobolev embedding, we have

$$
\left(\int_{\mathbb{R}^{N}}\left|y\left(\omega_{L, n}\right)\right|^{2^{\sharp}}\right)^{\frac{2}{2 \sharp}} \leq C \int_{\mathbb{R}_{+}^{N+1}}\left|\nabla \omega_{L, n}\right|^{2} d x d y,
$$

where constant $C>0$, see for example [39]. So combining (4.4) and (4.5), we have

$$
\begin{aligned}
& \left(\int_{\mathbb{R}^{N}}\left|y\left(v_{L, n}^{\beta-1}\left|v_{n}\right|\right)\right|^{2^{\sharp}}\right)^{\frac{2}{2 \sharp}} \\
& \quad \leq C \beta\left(\left(\varepsilon+V_{0}\right) \int_{\mathbb{R}^{N}}\left|y\left(v_{L, n}\right)\right|^{2(\beta-1)}\left|y\left(v_{n}\right)\right|^{2} d x+C_{\varepsilon} \int_{\mathbb{R}^{N}}\left|y\left(v_{L, n}\right)\right|^{2(\beta-1)}\left|y\left(v_{n}\right)\right|^{2^{\sharp}} d x\right),
\end{aligned}
$$

for constant $C>0$.

Next, we claim $\left|y\left(\left|v_{n}\right|\right)\right| \in L^{\frac{\left(2^{\sharp}\right)^{2}}{2}}\left(\mathbb{R}^{N}\right)$. In fact, choosing $\beta=\frac{2^{\sharp}}{2}$ in (4.6) and using Hölder inequality, we have

$$
\begin{aligned}
& \left(\int_{\mathbb{R}^{N}}\left|y\left(v_{L, n}^{\frac{2^{\sharp}-2}{2}}\left|v_{n}\right|\right)\right|^{2^{\sharp}} d x\right)^{\frac{2}{2^{\sharp}}} \\
& \leq C \beta\left(\left(\varepsilon+V_{0}\right) \int_{\mathbb{R}^{N}}\left|y\left(v_{L, n}\right)\right|^{2^{\sharp}-2}\left|y\left(v_{n}\right)\right|^{2} d x+C_{\varepsilon} \int_{\mathbb{R}^{N}}\left|y\left(v_{L, n}\right)\right|^{2^{\sharp}-2}\left|y\left(v_{n}\right)\right|^{2^{\sharp}} d x\right) \\
& \leq C \beta\left(\varepsilon+V_{0}\right) \int_{\mathbb{R}^{N}}\left|y\left(v_{L, n}\right)\right|^{2^{\sharp}-2}\left|y\left(v_{n}\right)\right|^{2} d x \\
& +C C_{\varepsilon} \beta\left(\int_{\mathbb{R}^{N}}\left(\left|y\left(v_{L, n}\right)\right|^{\frac{2^{\sharp}-2}{2}}\left|y\left(v_{n}\right)\right|\right)^{2^{\sharp}} d x\right)^{\frac{2}{2 \sharp}}\left(\int_{\mathbb{R}^{N}}\left(\left|y\left(v_{n}\right)\right|\right)^{2^{\sharp}} d x\right)^{\frac{2^{\sharp}-2}{2^{\sharp}}} .
\end{aligned}
$$

Choosing proper $\varepsilon>0$, we can obtain

$$
\left(\int_{\mathbb{R}^{N}}\left|y\left(v_{L, n}^{\frac{2^{\sharp}-2}{2}}\left|v_{n}\right|\right)\right|^{2^{\sharp}} d x\right)^{\frac{2}{2 \sharp}} \leq C \int_{\mathbb{R}^{N}}\left|y\left(v_{L, n}\right)\right|^{2^{\sharp}-2}\left|y\left(v_{n}\right)\right|^{2} d x,
$$


for positive constant $C$. Let $L \rightarrow+\infty$, it yields

$$
\left(\int_{\mathbb{R}^{N}}\left|y\left(\left|v_{n}\right|\right)\right|^{\frac{\left(2^{\sharp}\right)^{2}}{2}} d x\right)^{\frac{2}{2^{\sharp}}} \leq C \int_{\mathbb{R}^{N}}\left|y\left(v_{n}\right)\right|^{2^{\sharp}} d x<+\infty .
$$

Now we let

$$
t=\frac{\left(2^{\sharp}\right)^{2}}{2(2 \sharp-2)},
$$

it follows that $2 t /(t-1)<2^{\sharp}$. We estimate the right-and side of (4.6). By Hölder inequality

$$
\begin{aligned}
\int_{\mathbb{R}^{N}}\left|y\left(v_{L, n}\right)\right|^{2(\beta-1)}\left|y\left(v_{n}\right)\right|^{2^{\sharp}} d x & \leq\left(\int_{\mathbb{R}^{N}}\left|y\left(v_{n}\right)\right|^{\left(2^{\sharp}-2\right) t} d x\right)^{\frac{1}{t}}\left(\int_{\mathbb{R}^{N}}\left|y\left(v_{n}\right)\right|^{\frac{2 \beta t}{f-1}} d x\right)^{\frac{t-1}{t}} \\
& \leq C\left(\int_{\mathbb{R}^{N}}\left|y\left(v_{n}\right)\right|^{\frac{2 \beta t}{t-1}} d x\right)^{\frac{t-1}{t}} .
\end{aligned}
$$

On the other hand, set $a=\frac{2^{\sharp}\left(2^{\sharp}-1\right)}{2(\beta-1)}$ and $b=2 \beta-a$, we see that $a, b \in\left(0,2^{\sharp}\right)$.Then by Young's inequality, we have

$$
\begin{aligned}
\int_{\mathbb{R}^{N}}\left|y\left(v_{n}\right)\right|^{2 \beta} d x & \leq \frac{a}{2 \sharp} \int_{\mathbb{R}^{N}}\left|y\left(v_{n}\right)\right|^{2^{\sharp}} d x+\frac{2^{\sharp}-a}{2 \sharp} \int_{\mathbb{R}^{N}}\left|y\left(v_{n}\right)\right|^{\frac{2^{\sharp} b}{2^{-}-a}} d x \\
& \leq C\left(\int_{\mathbb{R}^{N}}\left|y\left(v_{n}\right)\right|^{2^{\sharp}} d x+\int_{\mathbb{R}^{N}}\left|y\left(v_{n}\right)\right|^{2 \beta-2+2^{\sharp}} d x\right),
\end{aligned}
$$

for $C>0$.

Combining (4.6), (4.8) and (4.9), we have

$$
\left(\int_{\mathbb{R}^{N}}\left|y\left(v_{L, n}^{\beta-1}\left|v_{n}\right|\right)\right|^{2^{\sharp}}\right)^{\frac{2}{2 \sharp}} \leq C \beta^{2}\left(\int_{\mathbb{R}^{N}}\left|y\left(v_{n}\right)\right|^{\frac{2 \beta t}{t-1}} d x\right)^{\frac{t-1}{t}},
$$

namely,

$$
\left(\int_{\mathbb{R}^{N}}\left|y\left(\left|v_{n}\right|\right)\right|^{\beta 2^{\sharp}}\right)^{\frac{1}{\beta}} \leq C^{\frac{2^{\sharp}}{2 \beta}} \beta^{\frac{2^{\sharp}}{\beta}}\left(\int_{\mathbb{R}^{N}}\left|y\left(v_{n}\right)\right|^{\frac{2 \beta t}{t-1}} d x\right)^{\frac{(t-1) 2^{\sharp}}{2 t \beta}} .
$$

For $i \geq 0$, we define $\beta_{i+1}$ inductively so that

$$
\frac{2 \beta_{i+1} t}{t-1}=\beta_{i} 2^{\sharp},
$$

that is,

$$
\beta_{i+1}=\frac{2^{\sharp}(t-1)}{2 t} \beta_{i},
$$

and $\beta_{1}=2^{\sharp} / 2$. Therefore, we have

$$
\left(\int_{\mathbb{R}^{N}}\left|y\left(\left|v_{n}\right|\right)\right|^{\beta_{i+1} 2^{\sharp}}\right)^{\frac{1}{\beta_{i+1}}} \leq C^{\frac{22^{\sharp}}{2 \beta_{i+1}}} \beta_{i+1}^{\frac{2^{\sharp}}{\beta_{i+1}}}\left(\int_{\mathbb{R}^{N}}\left|y\left(v_{n}\right)\right|^{\beta_{i} 2^{\sharp}} d x\right)^{\beta_{i}} .
$$

Let

$$
A_{i}=\left(\int_{\mathbb{R}^{N}}\left|y\left(v_{n}\right)\right|^{\beta_{i} 2^{\sharp}} d x\right)^{\beta_{i}} .
$$


Iterating by (4.11), w obtain

$$
A_{i+1} \leq \Pi_{m=2}^{i+1} C^{\frac{2 \sharp}{2 \beta m}} \beta_{m}^{\frac{2^{\sharp}}{\beta_{m}}} A_{1} \leq C_{0} A_{1} \text {. }
$$

This implies that

$$
\left\|y\left(\left|v_{n}\right|\right)\right\|_{L^{\infty}\left(\mathbb{R}^{N)}\right.} \leq C_{0} A_{1} .
$$

We complete the proof by using the fact

$$
A_{1}=\left(\int_{\mathbb{R}^{N}}\left|y\left(v_{n}\right)\right|^{\frac{\left(2^{\sharp}\right)^{2}}{2}} d x\right)^{\frac{2}{2^{\sharp}}}<\infty .
$$

By a standard arguments as Proposition 2.5 in [20] and Theorem 7.1 in [40], we can prove that $y\left(v_{n}\right)$ is exponential decay and we omit the details here.

We are now ready to prove the main result of this paper.

Proof of Theorem 1.1. We fix a small $\delta>0$ such that $M_{\delta} \subset \mathcal{O}$. We first claim that there exists some $\tilde{\varepsilon}_{\delta}>0$ such that for any $\varepsilon \in\left(0, \tilde{\varepsilon}_{\delta}\right)$ and any solution $w_{\varepsilon} \in \widetilde{\mathcal{N}}_{\varepsilon}$ of problem (2.10), there holds

$$
\left\|y\left(w_{\varepsilon}\right)\right\|_{L^{\infty}\left(\mathbb{R}^{N} \backslash \mathcal{O}_{\varepsilon}\right)}<a .
$$

We prove this claim by a contradiction argument and suppose that, there exists subsequence $\varepsilon_{n} \rightarrow 0^{+}, w_{n}:=$ $w_{\varepsilon_{n}} \in \widetilde{\mathcal{N}}_{\varepsilon_{n}}$ such that $\mathcal{J}_{\varepsilon_{n}}^{\prime}\left(w_{n}\right)=0$ and

$$
\left\|y\left(w_{n}\right)\right\|_{L^{\infty}\left(\mathbb{R}^{N} \backslash \mathcal{O}_{\varepsilon_{n}}\right)} \geq a .
$$

By Lemma 4.1, we know $\mathcal{J}_{\mathcal{E}_{n}}\left(w_{n}\right) \rightarrow c_{0}$ and thus we can invoke Proposition 3.7 to obtain a sequence $\tilde{z}_{n} \subset \mathbb{R}^{N}$ such that $\varepsilon_{n} \tilde{z}_{n} \rightarrow z_{0} \in M$.

If we take $r>0$ such that $B_{r}\left(z_{0}\right) \subset B_{2 r}\left(z_{0}\right) \subset \mathcal{O}$ we have

$$
B_{r / \varepsilon_{n}}\left(z_{0} / \varepsilon_{n}\right)=\frac{1}{\varepsilon_{n}} B_{r}\left(z_{0}\right) \subset \mathcal{O}_{\varepsilon_{n}} .
$$

Moreover, for any $z \in B_{r / \varepsilon_{n}}\left(\tilde{z}_{n}\right)$, there holds

$$
\left|z-\frac{z_{0}}{\varepsilon_{n}}\right| \leq\left|z-\tilde{z}_{n}\right|+\left|\tilde{z}_{n}-\frac{z_{0}}{\varepsilon_{n}}\right|<\frac{1}{\varepsilon_{n}}\left(r+o_{n}(1)\right)<\frac{2 r}{\varepsilon_{n}},
$$

for $n$ large. For this values of $n$ we have that $B_{r / \varepsilon_{n}}\left(\tilde{z}_{n}\right) \subset \mathcal{O}_{\varepsilon_{n}}$ or ,equivalently, $\mathbb{R}^{N} \backslash \mathcal{O}_{\varepsilon_{n}} \subset \mathbb{R}^{N} \backslash B_{r / \varepsilon_{n}}\left(\tilde{z}_{n}\right)$.

On the other hand, it follows from Lemma 4.1, there is a $R>0$ such that

$$
y\left(w_{n}\right)<a, \quad \text { for }|x| \geq R \text { and } \forall n \in \mathbb{N},
$$

for where it follows that

$$
y\left(w_{n}(x, \cdot)\right)=y\left(v_{n}\left(x-\tilde{z}_{n}, \cdot\right)\right)=y\left(w_{n}\left(x-\tilde{z}_{n}, \cdot\right)\right)<a
$$

for $x \in B_{R}^{c}\left(\tilde{z}_{n}\right)$ and $n \in \mathbb{N}$. Since there exists $n_{0} \in \mathbb{N}$ such that for any $n \geq n_{0}$ and $r / \varepsilon_{n}>R$, there holds

$$
\mathbb{R}^{N} \backslash \mathcal{O}_{\varepsilon_{n}} \subset \mathbb{R}^{N} \backslash B_{r / \varepsilon_{n}}\left(\tilde{z}_{n}\right) \subset \mathbb{R}^{N} \backslash B_{R}\left(\tilde{z}_{n}\right) .
$$

Therefore, there holds

$$
y\left(w_{n}\right)<a, \quad \forall \mathbb{R}^{N} \backslash \mathcal{O}_{\varepsilon_{n}},
$$

which contradicts to (4.13) and the claim holds true.

Let $\hat{\varepsilon}_{\delta}$ given by Theorem 3.9 and let $\varepsilon_{\delta}:=\min \left\{\hat{\varepsilon}_{\delta}, \tilde{\varepsilon}_{\delta}\right\}$. We will prove the theorem for this choice of $\varepsilon_{\delta}$. Let $\varepsilon \in\left(0, \varepsilon_{\delta}\right)$ be fixed. By using Theorem 3.9 we can get $c a t_{M_{\delta}}(M)$ nontrivial solutions of (2.10). If $w \in$ $H_{A_{\varepsilon}}^{1}\left(\mathbb{R}_{+}^{N+1}, \mathbb{C}\right)$ is one of these solutions, we have that $w \in \widetilde{\mathcal{N}}_{\varepsilon}$ and we can use (4.14) and the definition of $g$ to conclude that $g(\cdot,|y(w)|)=f(|y(w)|)$. Hence, $u(x)=y(w(x, y))$ is also a solution of problem (2.6). By an easy 
calculation we see that $v(x):=u(x / \varepsilon)$ is a solution of the original problem (1.2). Then problem (1.2) has at least $c a t_{M_{\delta}}(M)$ nontrivial solutions.

Now we consider $\varepsilon_{n} \rightarrow 0^{+}$and take a sequence $w_{n} \in H_{A_{\varepsilon_{n}}}^{1}\left(\mathbb{R}_{+}^{N+1}, \mathbb{C}\right)$ of solutions of problem (2.10) as above. In order to study the behavior of the maximum points of $u_{n}=y\left(w_{n}\right)$, we first note that, by the definition of $g$ and $(f 1)-(f 2)$, there exists $0<\tau<a$ small such that

$$
g\left(\varepsilon_{n} x, s\right) s^{2}=f(s) s^{2} \leq \frac{V_{0}}{K} s^{2}
$$

for all $x \in \mathbb{R}^{N}$ and $s \leq \tau$.

Using a similar argument as above, we can take $R>0$ such that

$$
\left\|u_{n}\right\|_{L^{\infty}\left(B_{R}^{c}\left(\tilde{z}_{n}\right)\right)}<\tau .
$$

Up to subsequence, we may also assume that

$$
\left\|u_{n}\right\|_{L^{\infty}\left(B_{R}\left(\tilde{z}_{n}\right)\right)}>\tau .
$$

Otherwise, if this is not the case, we have

$$
\left\|u_{n}\right\|_{L^{\infty}\left(B_{R}\left(\tilde{z}_{n}\right)\right)} \leq \tau,
$$

and so it follows from $\mathcal{J}_{\mathcal{E}_{n}}^{\prime}\left(w_{n}\right)=0$, (4.15) and take a same calculation as (2.17)-(2.18), we can get a contradiction. Therefore, (4.17) holds.

By observing (4.16) and (4.17), we see that the maximum points $p_{n} \in \mathbb{R}^{N}$ of $u_{n}$ belongs to $\left.B_{R}\left(\tilde{z}_{n}\right)\right)$. Hence $p_{n}=\tilde{z}_{n}+q_{n}$ for some $q_{n} \in B_{R}(0)$. Recalling that the associated solution of (1.2) is of the form $v_{n}(x):=u_{n}\left(x / \varepsilon_{n}\right)$, we conclude that the maximum point $\eta_{\varepsilon_{n}}$ of $v_{n}$ is $\eta_{\varepsilon_{n}}:=\varepsilon_{n} \tilde{z}_{n}+\varepsilon_{n} q_{n}$. Since $\left\{q_{n}\right\} \subset B_{R}(0)$ is bounded and $\varepsilon_{n} \tilde{z}_{n} \rightarrow z_{0} \in M$ (according Proposition 3.7), we obtain

$$
\lim _{n \rightarrow \infty} V\left(\eta_{\varepsilon_{n}}\right)=V\left(z_{0}\right)=-V_{0}
$$

Acknowledgments: The author would like to express his thanks to Prof. Jianfu Yang for his valuable comments and suggestions. This article is supported by the Fund of Jiangxi Provincial Department of Education (No. GJJ160335), the NSFC (Nos. 11701239 and 11871253) and the Program for Cultivating Youths of Outstanding Ability in Jiangxi Normal University.

\section{References}

[1] T. Ichinose, Magnetic relativistic Schrödinger operators and imaginary-time path integrals, in: Mathematical Physics, Spectral Theory and Stochastic Analysis, in: Oper. Theory Adv. Appl., vol. 232, Birkhäuser/Springer, Basel AG, Basel, 2013, pp. 247-297.

[2] T. Ichinose and H. Tamura, Imaginary-time path integral for a relativistic spinless particle in an electromagnetic field, Comm. Math. Phys. 105 (1986) 239-257.

[3] V. Iftimie, M. Mantoiu and R. Purice, Magnetic pseudodifferential operators. Publ. Res. Inst. Math. Sci. 43 (2007), no. 3 , 585-623.

[4] R. Frank, E. Lieb and R. Seiringer, Hardy-Lieb-Thirring inequalities for fractional Schrödinger operators. J. Amer. Math. Soc. 21 (2008), no. 4, 925-950.

[5] E. Lieb and R. Seiringer, The stability of matter in quantum mechanics. Cambridge University Press, Cambridge, 2010.

[6] T. Umeda and M. Nagase, Spectra of relativistic Schrödinger operators with magnetic vector potentials. Osaka J. Math. 30 (1993), no. 4, 839-853.

[7] S. Cingolani and S. Secchi, Intertwining solutions for magnetic relativistic Hartree type equations. Nonlinearity 31 (2018), no. 5, 2294-2318.

[8] L. Caffarelli and L. Silvestre, An extension problem related to the fractional Laplacian. Comm. Partial Differential Equations 32 (2007), no. 7-9, 1245-1260. 
[9] A. Elgart and B. Schlein, Mean field dynamics of boson stars. Comm. Pure Appl. Math. 60 (2007), no. 4, 500-545.

[10] J. Fröhlich, B. Jonsson and E. Lenzmann, Boson stars as solitary waves. Comm. Math. Phys. 274 (2007), no. 1, 1-30.

[11] E. Lieb and H. Yau, The Chandrasekhar theory of stellar collapse as the limit of quantum mechanics. Comm. Math. Phys. 112 (1987), no. 1, 147-174.

[12] Y. Cho, and T. Ozawa, On the semirelativistic Hartree-type equation. SIAM J. Math. Anal. 38 (2006), no. 4, 1060-1074.

[13] J. Fröhlich and E. Lenzmann, Dynamical collapse of white dwarfs in Hartree- and Hartree-Fock theory. Comm. Math. Phys. 274 (2007), no. 3, 737-750.

[14] E. Lenzmann, Uniqueness of ground states for pseudorelativistic Hartree equations. Anal. PDE 2 (2009), no. 1, 1-27.

[15] M. Esteban and P.L. Lions, Stationary solutions of nonlinear Schrödinger equations with an external magnetic field, in: Partial Differential Equations and the Calculus of Variations, vol. I, in: Progr. Nonlinear Differential Equations Appl., vol. 1, Birkhäuser Boston, Boston, MA, 1989, pp. 401-449.

[16] K. Kurata, Existence and semi-classical limit of the least energy solution to a nonlinear Schrödinger equation with electromagnetic fields, Nonlinear Anal. 41 (2000) 763-778.

[17] S. Cingolani, Semiclassical stationary states of nonlinear Schrödinger equations with an external magnetic field, J. Differential Equations 188 (2003) 52-79.

[18] C.O. Alves, G.M. Figueiredo and M.F. Furtado, Multiple solutions for a nonlinear Schrödinger equation with magnetic fields, Comm. Partial Differential Equations 36 (2011) 1565-1586.

[19] G. Arioli and A. Szulkin, A semilinear Schrödinger equation in the presence of a magnetic field, Arch. Ration. Mech. Anal. 170 (2003) 277-295.

[20] J. Chabrowski, and A. Szulkin, On the Schrödinger equation involving a critical Sobolev exponent and magnetic field. Topol. Methods Nonlinear Anal. 25 (2005), no. 1, 3-21.

[21] S. Cingolani and S. Secchi, Semiclassical states for NLS equations with magnetic potentials having polynomial growths, J. Math. Phys. 46 (2005) 053503, 19 pp.

[22] V. Ambrosio and P. d'Avenia, Nonlinear fractional magnetic Schrödinger equation: Existence and multiplicity, J. Differential Equations 264 (2018) 3336-3368.

[23] A. Fiscella, A. Pinamonti and E. Vecchi, Multiplicity results for magnetic fractional problems, J. Differential Equations 263 (2017) 4617-4633.

[24] B. Zhang, M. Squassina and Z. Xia, Fractional NLS equations with magnetic field, critical frequency and critical growth. Manuscripta Math. 155 (2018), no. 1-2, 115-140.

[25] S. Mao and A. Xia, Multiplicity results of nonlinear fractional magnetic Schrödinger equation with steep potential. Appl. Math. Lett. 97 (2019), 73-80.

[26] P. d’Avenia and M. Squassina, Ground states for fractional magnetic operators, ESAIM Control Optim. Calc. Var. 24 (2018), 1-24.

[27] A. Bahrouni, H. Ounaies and V.D. Rădulescu, Infinitely many solutions for a class of sublinear Schrödinger equations with indefinite potentials. Proc. Roy. Soc. Edinburgh Sect. A 145 (2015), no. 3, 445-465.

[28] A. Bahrouni, H. Ounaies and V.D. Rădulescu, Bound state solutions of sublinear Schrödinger equations with lack of compactness. Rev. R. Acad. Cienc. Exactas Fis. Nat. Ser. A Mat. RACSAM 113 (2019), no. 2, 1191-1210.

[29] Y.F. Xue and C.L. Tang, Existence of a bound state solution for quasilinear Schrödinger equations. Adv. Nonlinear Anal. 8 (2019), no. 1, 323-338.

[30] M. del Pino and F. Felmer, Local mountain passes for semilinear elliptic problems in unbounded domains. Calc. Var. Partial Differential Equations 4 (1996), no. 2, 121-137.

[31] J. Moser, A new proof of Giorgi's theorem concerning the regularity problem for elliptic differential equations. Commun. Pure Appl. Math. 13, 457-468 (1960).

[32] A. Szulkin and T. Weth, The method of Nehari manifold. In: Gao, D.Y., Montreanu, D. (eds.) Handbook of Nonconvex Analysis and Applications, pp. 2314-2351. Inernational Press, Boston (2010).

[33] X. He and W. Zou, Existence and concentration result for the fractional Schrödinger equations with critical nonlinearities. Calc. Var. Partial Differential Equations 55 (2016), no. 4, Art. 91, 39 pp.

[34] X. He and W. Zou, Multiplicity of concentrating positive solutions for Schrödinger-Poisson equations with critical growth. Nonlinear Anal. 170 (2018), 142-170.

[35] M. Willem, Minimax Theorems. Birckhäuser, Boston (1996).

[36] P. L. Lions, The concentration-compactness principle in the calculus of variations. The locally compact case. II. Ann. Inst. H. Poincaré Anal. Non Linéaire 1 (1984), no. 4, 223-283.

[37] I. Ekeland, On the variational principle, J. Math. Anal. Appl. 17 (1974) 324-353.

[38] A. Xia and J. Yang, Regularity of nonlinear equations for fractional Laplacian. Proc. Amer. Math. Soc. 141 (2013), no. 8, 2665-2672.

[39] X. Cabré, and J. Tan, Positive solutions of nonlinear problems involving the square root of the Laplacian. Adv. Math. 224, 2052-2093 (2010).

[40] S. Cingolani and S. Secchi, Ground states for the pseudo-relativistic Hartree equation with external potential. Proc. Roy. Soc. Edinburgh Sect. A 145 (2015), no. 1, 73-90. 